\title{
Applications and Therapeutic Actions of Complementary and Alternative Medicine for Women with Genital Infection
}

\author{
Chenfang Liu, ${ }^{1}$ Yuehui Zhang, ${ }^{2,3,4}$ Sai Kong, ${ }^{1}$ Ilene Tsui, ${ }^{4}$ Yang Yu, ${ }^{1}$ and Fengjuan Han ${ }^{2}$ \\ ${ }^{1}$ Department of Obstetrics and Gynecology, Heilongjiang University of Chinese Medicine, Harbin, \\ Heilongjiang 150040, China \\ ${ }^{2}$ Department of Obstetrics and Gynecology, First Affiliated Hospital, Heilongjiang University of Chinese Medicine, \\ Harbin, Heilongjiang 150040, China \\ ${ }^{3}$ Center for Post-Doctoral Studies, Heilongjiang University of Chinese Medicine, Harbin 150040, China \\ ${ }^{4}$ Pennsylvania State University College of Medicine, Hershey, PA 17033, USA
}

Correspondence should be addressed to Fengjuan Han; hanfengjuan2004@163.com

Received 9 September 2013; Accepted 16 December 2013; Published 4 February 2014

Academic Editor: Xiaoke Wu

Copyright (c) 2014 Chenfang Liu et al. This is an open access article distributed under the Creative Commons Attribution License, which permits unrestricted use, distribution, and reproduction in any medium, provided the original work is properly cited.

\begin{abstract}
Genital infection is a common worldwide disease among females with clinical features such as bilateral lower abdominal tenderness, abnormal vaginal or cervical discharge, fever, abnormal vaginal bleeding, dyspareunia, vaginal itching, and adnexal tenderness, which can significantly impair women's health and quality of life. Genital infection is commonly treated with antibiotics, leading to an imbalance in gut flora due to prolonged use of antibiotics. Therefore, it is necessary to discover safe and efficacious alternative treatment strategies for patients with genital infection. Complementary and alternative medicine (CAM) is becoming increasingly prevalent among women with genital infection. CAM has interested the western mainstream medical community because of its less invasive, safe, effective, economical, and convenient therapies. CAM focuses on the prevention and treatment of disease and has become an important force in treating chronic disease. During the last few decades, the popularity of CAM has gradually increased. To further understand the efficacy of CAM in treating genital infection, our paper will review the current progress of treating genital infection including vulvitis, vaginitis, cervicitis, and pelvic inflammatory disease (PID) with CAM therapies. Several CAM strategies including traditional Chinese medicine (TCM), acupuncture, Psychology interference, and physical therapy are introduced in this review.
\end{abstract}

\section{Introduction}

Genital infection is common among women of all ages and includes a variety of different diseases of the genital tract including vulvitis, vaginitis, cervicitis, and pelvic inflammatory disease (PID). Infection plays an important role in gynecology and infertility, affecting the ovary, uterus; and the embryo and implantation [1]. Long-term and repeated infections will cause damage and adhesion of tubal mucosa, eventually leading to infertility. Due to the nature of these wide-ranging effects, it is important to find effective therapies for genital infection. Currently, genital infection is commonly treated with antibiotics. As a side effect, the increased drugresistance has reduced therapeutic efficacy. CAM is defined as the complement for conventional medical therapy, providing diagnosis, therapy, and prevention that conventional medical regimens otherwise cannot treat. CAM mainly contains the following methods: (i) alternative medical system: TCM, Ayurveda, and homeopathy; (ii) mind-body intervention: meditation and biofeedback; (iii) biologically based therapies: herbal therapy and special diet therapy; (iv) manipulative and body-based methods: chiropractic and massage; (v) energy therapies [2]. At present, several CAM methods have been used in treating genital infection. This review briefly 
summarizes the current progress of treating genital infection with CAM and introduces the potential mechanisms.

\section{TCM Utilization in Genital Infection}

As a form of primary care throughout many Asian countries, TCM is an important part of CAM. The basic TCM therapies include Chinese herbal medicine (CHM) and acupuncture. Chinese herbs and acupuncture are important components of TCM. They have been used for disease treatment and prevention and as alternative therapies for over 2000 years [3]. TCM has significant advantages in treating genital infection. In TCM, genital infection is usually classified as the area of "leukorrheal diseases," "the woman abdominal pain," and "abdominal mass" and the treatment is commonly based on syndrome differentiation. CHM acts on "Zang-fu" viscera internally and part of skin externally, while acupuncture works by stimulating certain areas of the external body. Indeed, a number of articles published within the last decade have examined the use of TCM for the treatment of genital infection.

2.1. The Effect of Chinese Herbs on Genital Infection. Based on TCM theory of syndrome differentiation, genital infection is divided into several TCM syndrome types, and then different traditional Chinese medicines are composed together to treat different syndrome types. Two approaches can be used in treatment: oral and external approaches, which can be applied independently or in combination. According to the theory of TCM, the pathogen of genital infection can be summarized as follows: heat, toxin, damp, stasis, and cold. The accumulation of these factors in the genitals leads to pathological changes, causing the symptoms of genital infection.

\subsubsection{Oral Administration of CHM for Genital Infection in} TCM. Clinical research has shown that Chinese medicinal compounds have certain satisfactory effects on genital infection including vulvitis, vaginitis, cervicitis, and PID. These results were used to evaluate whether Chinese herbs could be used as a complementary drug to treat specific symptoms. Among all the formulae studied for various genital inflammatory diseases, "Shaofuzhuyutang" et al. (Table 1) were the most frequently used formulas and demonstrated a higher effect on reducing genital infectious diseases.

\subsubsection{External Therapy to Genital Infection in TCM. External} therapy of TCM is a form of traditional medicine therapy, preventing and curing diseases by stimulating the meridians, acupuncture points, skin, mucous membranes, muscles, and bones. External therapy has experienced a long history, dating back to ancient China, and gradually developed into a unique medical approach. There are two main approaches in external therapy. (1) Skin penetration and mucosal absorption: there are many ways of transdermal administration with Chinese medicine, such as dressing, stickers, steaming, washing, and bathing. Most drugs are easily absorbed from the dermis to the human body after passing through the skin. The greatest advantage of transdermal administration is that it avoids potentially damaging the gastrointestinal tract and liver while also avoiding metabolic effects of these respective organs, thereby increasing drug effect and maintaining plasma concentration over a sustained period of time [33]. (2) Mucosal absorption: the route of administration is through the mucosa, including mouth, eyes, nose, genitalia, and anus. We have reviewed several related studies that verify the efficacy of CHM as external therapy for various genital infectious diseases (Table 2).

(i) Rectal Administration. Currently, rectal administration includes retention enema and rectal infusion with liquid Chinese herbal medicine [52]. Chronic pelvic inflammatory disease (CPID) mainly spreads inside the pelvic cavity, while the rectum neighbors both the uterus and uterine adnexa, making rectal administration a plausible method of drug delivery. There are dense veins in female pelvic tissues and organs with rich blood supply. Since the rectal mucosa is relatively thin, the medicine will directly act on the pelvic cavity at a high concentration. Liquid Chinese herbal medicine by rectal administration was shown to be a good treatment for CPID. This method not only promotes blood circulation, improves tissue nutrition, and reduces the inflammatory exudate but also inhibits the proliferation of connective tissues, thereby promoting the absorption of the inflammatory mass, releasing tissue adhesions and relieving local spasms [53]. Further, retention enema also avoids the hepatic first-pass effect, reducing the burden on the liver.

(ii) Steaming Washing Therapy. Steaming washing therapy is a kind of traditional Chinese medicine therapy, where patients are usually told to take a hip bath with hot liquid herbal medicine. Before washing the certain area with the liquid, the steam can be absorbed to a certain degree. Both Chinese herbal compounds and the heat coordinate to accelerate the absorption of the medicine into the certain location [54].

(iii) Vaginal Lavage. Vaginal lavage is commonly used to treat vaginitis and cervicitis with various functions of cleaning the vagina, promoting vaginal blood circulation, reducing vaginal secretions, and relieving local hyperemia. Vaginal lavage with Chinese medicine serves two purposes: (1) to change the $\mathrm{pH}$ value within the vagina and inhibit or kill the Trichomonas bacteria; (2) to remove a large amount of vaginal secretions, thereby greatly reducing the number of pathogenic microorganisms. The clinical observation has shown satisfactory efficacy from the treatment of Trichomonas vaginitis by vaginal lavage with traditional Chinese medicine [55].

(iv) Intravaginal Administration. Some clinical observations reported that traditional Chinese medicine had achieved good effects on PID and vaginitis in treatment with the intravaginal-drug way. One study showed that the total effective rate was $98.2 \%$ treating PID in the vaginal fornix with the paste composed of Shuanghuanglian powder $0.6 \mathrm{~g}$, Huoxuezhitong scattered $3 \mathrm{~g}$, and Tetramethylpyrazine $2 \mathrm{~mL}$ [56]. 
TABLE 1: List of effective Chinese herbal compounds for genital infection with oral administration.

\begin{tabular}{|c|c|c|c|c|}
\hline $\begin{array}{l}\text { The frequency of } \\
\text { related articles }\end{array}$ & $\begin{array}{l}\text { Chinese herbal } \\
\text { compound }\end{array}$ & Formula composition & Disease & $\begin{array}{l}\text { The average effective } \\
\text { rate }\end{array}$ \\
\hline$N=41.38 \%[4-15]$ & Shaofuzhuyutang & $\begin{array}{l}\text { Foeniculum vulgare, Rhizoma } \\
\text { Zingiberis, Rhizoma Corydalis, } \\
\text { Commiphora molmol, Radix Angelicae } \\
\text { Sinensis, Ligusticum chuanxiong, } \\
\text { Cortex Cinnamomi, Radix Paeoniae } \\
\text { Rubra, Pollen Typhae, Faeces } \\
\text { Togopterori }\end{array}$ & PID & $\begin{array}{c}\mathrm{RR}, 1.23 \\
(1.17,1.28)\end{array}$ \\
\hline$N=10.34 \%[16-18]$ & Dangguishaoyaosan & $\begin{array}{l}\text { Radix Angelicae Sinensis, Chinese } \\
\text { herbaceous peony, Poria, } \\
\text { Atractylodes macrocephala, Rhizoma } \\
\text { Alismatis, Ligusticum chuanxiong }\end{array}$ & PID & $\begin{array}{c}\mathrm{RR}, 1.44 \\
(1.22,1.71)\end{array}$ \\
\hline$N=13.79 \%[19-22]$ & Guizhifulingtang & $\begin{array}{l}\text { Ramulus Cinnamomi, Poria, } \\
\text { Glycyrrhiza uralensis, Cortex Moutan, } \\
\text { Chinese herbaceous peony, Semen } \\
\text { Persicae }\end{array}$ & PID & $\begin{array}{c}\mathrm{RR}, 1.24 \\
(1.14,1.35)\end{array}$ \\
\hline$N=10.34 \%[23-25]$ & Danzhixiaoyaosan & $\begin{array}{l}\text { Atractylodes macrocephala, Radix } \\
\text { Bupleuri, Radix Angelicae Sinensis, } \\
\text { Poria, Glycyrrhiza uralensis, Cortex } \\
\text { Moutan, Gardenia jasminoides, } \\
\text { Chinese herbaceous peony }\end{array}$ & PID & $\begin{array}{c}\mathrm{RR}, 1.22 \\
(1.11,1.34)\end{array}$ \\
\hline$N=17.24 \%[26-30]$ & Bixieshenshitang & $\begin{array}{l}\text { Rhizoma Dioscoreae Collettii, Semen } \\
\text { Coicis, Rhizoma Smilacis Glabrae, } \\
\text { Pulvis Talci, Cortex Moutan, Rhizoma } \\
\text { Alismatis, Medulla Tetrapanacis, } \\
\text { Cortex Phellodendri }\end{array}$ & Vaginitis & $\begin{array}{c}\mathrm{RR}, 1.14 \\
(1.07,1.22)\end{array}$ \\
\hline$N=6.90 \%[31,32]$ & Wandaitang & $\begin{array}{l}\text { Atractylodes macrocephala, Rhizoma } \\
\text { Dioscoreae, Ginseng, Chinese } \\
\text { herbaceous peony, Semen Plantaginis, } \\
\text { Rhizoma Atractylodis, } \\
\text { Glycyrrhiza uralensis, Pericarpium } \\
\text { Citri Reticulatae, } \\
\text { Schizonepeta tenuifolia }\end{array}$ & Cervicitis/vaginitis & $\begin{array}{c}\mathrm{RR}, 1.37 \\
(1.22,1.54)\end{array}$ \\
\hline
\end{tabular}

(v) External Application. Grind the CHM into fine powder and put it in a warm bag. Applying the bag externally to the lower abdomen increases the drug concentration at certain focal points and allows direct absorption by heat conduction. External application helps to ameliorate blood circulation in the pelvic cavity and promote the absorption of inflammation [57]. Further, this method to a certain extent is able to avoid stimulating the gastric mucosa, so that the bioavailability of the drug can be prolonged.

\subsubsection{Single Herbs and Phytotherapy}

Single Herbs. Chinese medicine has various functions including heat-clearance, blood-activation, and stasis-elimination and acts as a general anti-inflammatory or analgesic and improves the immune function by focusing on the several pathogenic characteristics of the gynecological inflammation. Smilax is mainly used for the treatment of CPID and gynecological inflammation in clinic. An experimental study has shown that Smilax can inhibit the hyperplasia of endometrial inflammatory cells, promote the recovery of damaged epithelial cells, and reduce congestion and edema of serosa for CPID in rat models [58]. A pharmacodynamic screening found that the ethyl acetate extracted from Smilax was the effective fraction for CPID treatment with an antiinflammatory function. The anti-inflammatory substances of Smilax were ingredients of flavonoids, saponins, and tannins [59]. Comfrey oil, extracted from comfrey, which is Radix Lithospermi Root of Sinkiang Arnebia (a kind of Chinese herb medicine), was commonly used to treat candida vaginitis with a higher efficacy rate, lower recurrence, and minimal side effects. The effective ingredient of comfrey oil is Shikonin $\mathrm{C}_{16} \mathrm{H}_{16} \mathrm{O}_{5}$; it could be absorbed rapidly in the vagina, then alleviating or curing the symptoms after treating for 3 to 4 days, and the cure rate is 92\% [60]. Wild chrysanthemum suppository, extracted from the wild chrysanthemum, was proved to have a good effect on CPID by rectal administration with more rapid, sustained effects and less adverse effects [61]. Patrinia is a kind of perennial herb in Valerianaceae, containing a variety of saponins which inhibit a variety of bacterial and virus. Patrinia is mainly used to treat CPID due to a strong inhibitory effect on Staphylococcus aureus, Bacillus anthracis, Bacillus diphtheria, hepatitis B 
TABLE 2: List of effective Chinese herbal compounds for genital infection with external therapy of TCM.

\begin{tabular}{|c|c|c|c|c|c|}
\hline $\begin{array}{l}\text { The frequency of } \\
\text { related articles }\end{array}$ & $\begin{array}{l}\text { Chinese herbal } \\
\text { compound }\end{array}$ & Formula composition & Administration route & Disease & $\begin{array}{l}\text { The average } \\
\text { effective rate }\end{array}$ \\
\hline $\begin{array}{l}N=47.37 \% \\
{[34-42]}\end{array}$ & Hongtengtang & $\begin{array}{l}\text { Caulis Sargentodoxae, Herba } \\
\text { Patriniae, Herba Taraxaci, } \\
\text { Rhizoma Sparganii, Rhizoma } \\
\text { Curcumae, Radix Astragali }\end{array}$ & Rectal & PID & $\begin{array}{c}\mathrm{RR}, 1.31 \\
(1.23,1.39)\end{array}$ \\
\hline $\begin{array}{l}N=10.53 \% \\
{[43,44]}\end{array}$ & Kushentang & $\begin{array}{l}\text { Radix Sophorae Flavescentis, } \\
\text { Fructus Cnidii, Radix Angelicae } \\
\text { Dahuricae, Rhizoma } \\
\text { Atractylodis, Fructus Kochiae, } \\
\text { Cortex Phellodendri }\end{array}$ & $\begin{array}{c}\text { Vaginal } \\
\text { lavage/steaming } \\
\text { washing/intravaginal } \\
\text { administration }\end{array}$ & Vaginitis/cervicitis & $\begin{array}{l}\mathrm{RR}, 1.33 \\
(1.18,1.51)\end{array}$ \\
\hline $\begin{array}{l}N=15.79 \% \\
{[45-47]}\end{array}$ & Shechuangzisan & $\begin{array}{l}\text { Fructus Cnidii, Fructus Kochiae, } \\
\text { Radix Sophorae Flavescentis, } \\
\text { Stemona sessilifolia, Cortex } \\
\text { Phellodendri Chinensis, Cortex } \\
\text { Dictamni }\end{array}$ & $\begin{array}{c}\text { Steaming } \\
\text { washing/vaginal lavage }\end{array}$ & Vaginitis & $\begin{array}{c}\mathrm{RR}, 1.15 \\
(1.08,1.24)\end{array}$ \\
\hline $\begin{array}{l}N=26.32 \% \\
{[33,48-51]}\end{array}$ & Longdanxiegantang & $\begin{array}{l}\text { Radix Gentianae, Radix } \\
\text { Scutellariae, } \\
\text { Gardenia jasminoides Ellis, } \\
\text { Rhizoma Alismatis, Caulis } \\
\text { Akebiae, Semen Plantaginis, } \\
\text { Angelica sinensis, Radix } \\
\text { Rehmanniae, Radix Bupleuri, } \\
\text { Glycyrrhiza uralensis }\end{array}$ & Steaming washing & Vaginitis & $\begin{array}{l}\mathrm{RR}, 1.07 \\
(1.03,1.12)\end{array}$ \\
\hline
\end{tabular}

Streptococcus, Salmonella typhi, and Shigella [62]. Houttuynia cordata, drying part of the perennial herb Houttuynia (a kind of Chinese herb medicine), containing complex composition such as volatile oils, alkaloids, flavonoids, and polysaccharide which can inhibit a variety of bacteria and relieve pain and inflammation, has evidenced a good efficacy for CPID $[63,64]$. Some related studies have verified that some single herbs such as Cortex Moutan Radicis, Rhizoma Curcumae, and Radix Paeoniae can promote blood circulation and the inflammatory exudate absorption. In summary, single herb is an effective therapy for CPID and further deep clinical studies should be done in the near future. We have made a list of some frequent single herbs in treating various genital inflammatory diseases (Table 3), and they are also quite common in Chinese herbal compounds for genital infectious diseases.

Phytotherapy. Significant research has been done to evaluate the efficacy of some plants and their active extract against vaginal pathogens and has demonstrated that they could provide an effective approach for treatment of vaginitis [149]. Phytotherapy is mainly used to treat vaginitis with herbal medicines which are anticandida, antibacterial, and antiTrichomonas. These active extracts including carvacrol, 1,8cineole, geranial, germacrene-D, limonene, linalool, menthol, terpinen-4-ol, and thymol exhibit the beneficial effects on many types of vaginitis including bacterial vaginosis, vulvovaginal candidosis, and Trichomonas vaginitis. These extracts can block mycelial growth at a very low concentration according to human and animal studies [150] and may present a new direction for the future role of plants in treating vaginitis.
2.2. Mechanisms of Chinese Herbal Medicine in Treating Genital Infection. Modern medicine indicates that genital infection is mainly caused by the presence of pathogenic microorganisms, a declining immune system, or a pelvic microcirculation disorder [3]. Therefore, medication treatment is mainly focused on targeting pathogenic microorganisms, improving immunity, or promoting blood circulation. In recent years, some experimental studies have focused on the mechanism of treating genital infection with Chinese herbal compounds and Chinese herbal patents (Figure 1).

\subsubsection{Antibacterial Mechanism of Chinese Herbal Medicine.} In many cases, microorganisms from the vaginal and cervical flora are frequently associated with PID, including anaerobic and facultative bacteria, similar to those associated with bacterial vaginosis [151]. The laboratory study confirms that Chinese medicine has an inhibitory effect on certain pathogens. Ziying granule, which consists of Tokyo Violet Herb, Dandelion, and 7 other herbs, has a broad bactericidal effect on common pathogenic bacteria found in the pelvic cavity [152]. Penyanqing Granule, which consists of Amur Cork-Tree Bark, Paeonia suffruticosa, and several other herbs, has also been shown to be an effective Chinese herbal compound for the clinical treatment of PID. A study result has shown that the common pathogens of Escherichia coli and Staphylococcus aureus were inhibited by Penyanqing Granule in PID mice model and have an obvious dose-dependent effect [153]. Furthermore an experiment on the effect of different doses of Smilax on common gynecological bacterial infections has shown that it had the broadest antibacterial spectrum at the concentration of $2 \mathrm{~g} / \mathrm{mL}$, inhibiting S. aureus, 
Table 3: Frequent Chinese herbal medicine in internal and external therapies.

\begin{tabular}{|c|c|c|c|c|c|c|}
\hline & PID & $\begin{array}{c}\text { Frequency } \\
{[65-81]}\end{array}$ & Vaginitis & $\begin{array}{c}\text { Frequency } \\
{[82-93]}\end{array}$ & Cervicitis & $\begin{array}{c}\text { Frequency } \\
{[94-99]}\end{array}$ \\
\hline \multirow{9}{*}{$\begin{array}{l}\text { Frequent Chinese } \\
\text { herbal medicine in } \\
\text { oral administration o } \\
\text { CHM }\end{array}$} & Radix Paeoniae Rubra & $N=14$ & $\begin{array}{l}\text { Rhizoma Atractylodis } \\
\text { Macrocephalae }\end{array}$ & $N=10$ & $\begin{array}{l}\text { Rhizoma Atractylodis } \\
\text { Macrocephalae }\end{array}$ & $N=11$ \\
\hline & Sargentodoxa cuneata & $N=13$ & Dioscorea Opposita & $N=10$ & $\begin{array}{l}\text { Root of Chinese } \\
\text { Thorowax }\end{array}$ & $N=6$ \\
\hline & Cortex Moutan Radicis & $N=11$ & Plantago asiatica $\mathrm{L}$. & $N=8$ & Dioscorea Opposita & $N=5$ \\
\hline & Salvia miltiorrhiza & $N=11$ & Wolfiporia cocos & $N=7$ & Wolfiporia cocos & $N=5$ \\
\hline & f Ixeris denticulata & $N=11$ & $\begin{array}{l}\text { Root of Chinese } \\
\text { Thorowax }\end{array}$ & $N=7$ & White Pieony Root & $N=5$ \\
\hline & Herba Taraxaci & $N=9$ & $\begin{array}{l}\text { Cortex Phellodendri } \\
\text { Chinensis }\end{array}$ & $N=6$ & $\begin{array}{l}\text { Radix Codonopsis } \\
\text { Pilosulae }\end{array}$ & $N=4$ \\
\hline & Wolfiporia cocos & $N=9$ & $\begin{array}{l}\text { Radix Codonopsis } \\
\text { Pilosulae }\end{array}$ & $N=6$ & Rhizoma Atractylodis & $N=4$ \\
\hline & $\begin{array}{l}\text { Radix Angelicae } \\
\text { Sinensis }\end{array}$ & $N=9$ & $\begin{array}{l}\text { Pericarpium Citri } \\
\text { Reticulatae }\end{array}$ & $N=5$ & $\begin{array}{l}\text { Radix Angelicae } \\
\text { Sinensis }\end{array}$ & $N=4$ \\
\hline & PID & $\begin{array}{l}\text { Frequency } \\
{[100-116]}\end{array}$ & Vaginitis & $\begin{array}{l}\text { Frequency } \\
{[117-132]}\end{array}$ & Cervicitis & $\begin{array}{r}\text { Frequency } \\
{[133-148]}\end{array}$ \\
\hline \multirow{6}{*}{$\begin{array}{l}\text { Frequent Chinese } \\
\text { herbal medicine in } \\
\text { external therapy }\end{array}$} & Ixeris denticulate & $N=14$ & $\begin{array}{l}\text { Radix Sophorae } \\
\text { Flavescentis }\end{array}$ & $N=15$ & $\begin{array}{l}\text { Cortex Phellodendri } \\
\text { Chinensis }\end{array}$ & $N=11$ \\
\hline & Semen Persicae & $N=9$ & Fructus Cnidii & $N=14$ & Borneol & $N=10$ \\
\hline & Herba Taraxaci & $N=9$ & $\begin{array}{l}\text { Cortex Phellodendri } \\
\text { Chinensis }\end{array}$ & $N=12$ & $\begin{array}{l}\text { Radix Sophorae } \\
\text { Flavescentis }\end{array}$ & $N=10$ \\
\hline & Radix Paeoniae Rubra & $N=8$ & Stemona sessilifolia & $N=11$ & Fructus Cnidii & $N=7$ \\
\hline & Sargentodoxa cuneata & $N=8$ & Cortex Dictamni & $N=9$ & $\begin{array}{l}\text { Coptis chinensis } \\
\text { Franch }\end{array}$ & $N=7$ \\
\hline & Rhizoma Sparganii & $N=8$ & Fructus Kochiae & $N=7$ & Borax & $N=3$ \\
\hline
\end{tabular}

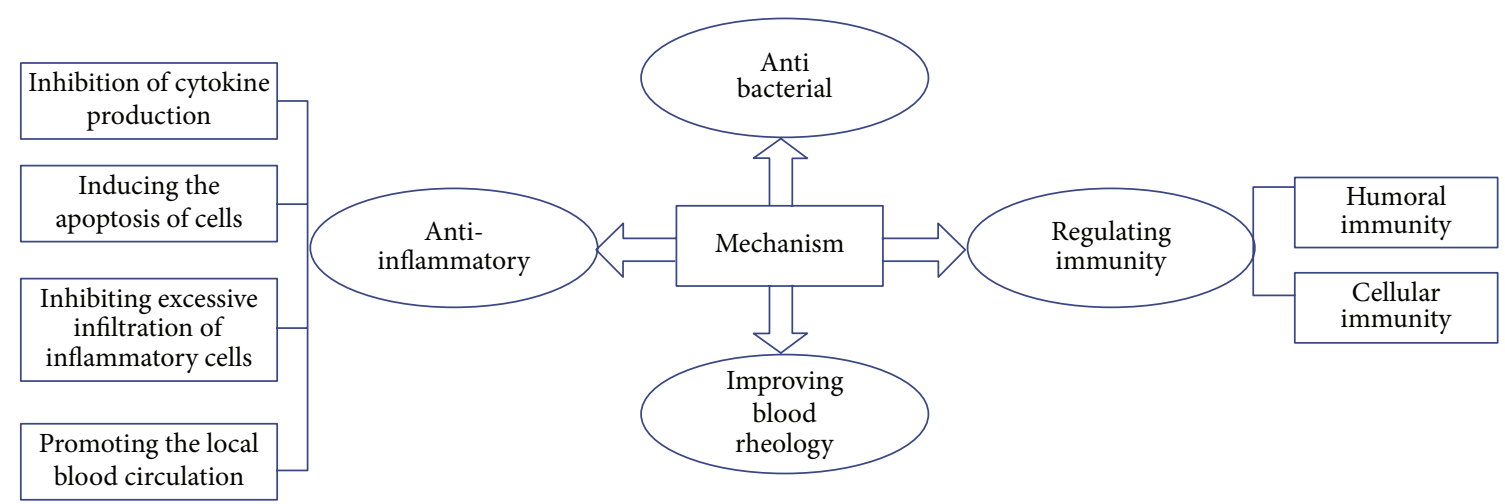

FIgure 1: Mechanisms of Chinese herbal medicine on genital infection.

E. coli, $P$. aeruginosa, and $P$. mirabilis significantly and regulating the vaginal $\mathrm{pH}$ value $[154,155]$. All of these therapies apply a promising alternative for treatment of genital infection.

\subsubsection{Anti-Inflammatory Mechanism of Chinese Herbal Med-} icine. Inflammation is a defense reaction between the body and various damaging factors. The inflammatory response depends on the nature and intensity of the pathogenic factor and the reactivity of the body [151]. The inflammatory response is primarily activated through a series of inflammatory mediators. The anti-inflammatory mechanism of certain
Chinese herbs can alleviate secondary tissue injury and eliminate or reduce inflammation by inhibiting the synthesis of inflammatory mediators, controlling the infiltration of inflammatory cells appropriately, and inducing apoptosis of infiltrated inflammatory cells.

(i) Inhibition of Cytokine Production. Chinese medicine may exhibit an anti-inflammatory effect by inhibiting the production of the cytokine secreted by activating lymphocytes, plasma cells, macrophages, and monocytes, such as IL-4 (interleukin-4) and TNF- $\alpha$ (tumor necrosis factor$\alpha$ ). TNF- $\alpha$ is one of thefirst appearing inflammatory fac- 
tors in the inflammatory response, which has a strong proinflammatory role in the early inflammatory response [156].

The clinical study showed that "Penqiangyan granule," which consisted of Salvia miltiorrhiza, Radix Paeoniae Rubra, Cuscuta chinensis, Pollen Typhae, and so forth (some kinds of Chinese herbal medicine), can significantly decrease the serum concentration of TNF- $\alpha$ with CPID patients to normal level. Furthermore, the experimental study found that the serum TNF- $\alpha$ concentration was higher in CPID model rat than the normal rat, while the serum TNF- $\alpha$ level was significantly decreased after treatment with "Penqiangyan granule" and reached normal level, which implied that the possible mechanism of "Penqiangyan granule" treatment on CPID was improving immune condition and inhibiting inflammatory cytokines [157]. Yan Ting, a kind of enemas of Chinese herbal medicine, consisting of Radix Paeoniae Rubra, Corydalis yanhusuo, Salvia miltiorrhiza, Sparganium stoloniferum, Rhizoma Curcumae, Sargentgloryvine Stem, and so forth, has proved that it could decrease the mRNA expression of TNF- $\alpha$ in fallopian tube, ease the adhesions and promote the recanalization of fallopian tube, and eventually improve the pregnancy rates in the rat model of salpingitis infertility [158].

(ii) Inducing the Apoptosis of Cells and Inhibiting Excessive Infiltration of Inflammatory Cells. CHM can induce cellular apoptosis, thereby reducing cell necrosis, effectively removing the inflammatory lesions of inflammatory cells and other proliferating cells, reducing secondary injury due to adjacent tissue cell necrosis, preventing inflammatory fibrous hyperplasia and scar. Furthermore, CHM can inhibit excessive infiltration of inflammatory cells by inhibiting hyperplasia of fibroblast and increasing degradation of fibrous tissue [159]. The activated nuclear factor $\mathrm{kB}$ (NF-KB) can inhibit or delay the apoptosis of polymorphonuclear (PMN), producing large amounts of cytokines which accelerated local and systemic inflammatory response. Caspase- 3 is a key enzyme in the downstream of apoptotic pathway; caspase-3 activated will promote apoptosis factors, ultimately leading to apoptosis through caspase-3-mediated signaling pathways, while CHM can induce apoptosis by regulating the expression of NF$\mathrm{KB}$ and caspase-3 to reduce the inflammatory response $[160,161]$. Study results suggested that the protein level of caspase- 3 was increased in the endometrium of CPID rat model with "Penyankang (a kind of compound of CHM consists (sic) of Caulis Sargentodoxae, Herba Patriniae, Salvia Miltiorrhiza and Sparganium Stoloniferum)" treatment than control group, which led to an increase of cell apoptosis, thereby reducing the inflammatory response [159]. Matrix metalloproteinase enzymes (MMP) play a major role in the process of extracellular matrix degradation, which can degrade almost all components of the extracellular matrix. The expression of MMP in the tissue of normal state is minimal, while it increases after the stimulation by inflammatory cytokines, hormones, and growth factors and also increases in the process of cell transformation [162]. The experimental results showed that the protein level of MMP had been enhanced after treatment with "Penyankang" in CPID rat model, which led to a degradation of the extracellular matrix, thereby reducing proliferation of the fibrous tissue in the chronic inflammation [160].

\subsubsection{The Fever-Reducing and Analgesic Effect of Chinese} Herbal Medicine. The great majority of women with PID are suffering from chronic pain, which can significantly influence their activities of daily life. Chronic pain management is an increasing challenge to the treatment for PID patients [163]. In recent years, many agents were commonly used to control chronic pain, such as nonsteroidal anti-inflammatory drugs (NSAIDs) and long-acting opiates, but NSAIDs have the hidden danger of serious toxicity, including gastrointestinal bleeding and renal failure, while the long-term efficacy of long-acting opiates has been poorly documented, and there is a significant potential for addiction and abuse [164166]. Obviously, new approaches are necessary for managing chronic pain, especially low-risk interventions with the potential to reduce the persistent pain.

Smilax is the rhizome of Smilax china L, belonging to Liliaceae plant. The preparations based on Smilax are widely used to treat CPID and other genial inflammations. A study found that the "Smilax capsules" can significantly inhibit the writhing response compared with the control group in PID mice model induced by acetic acid. Both of the higher and lower doses could significantly reduce mice writhing within 20 minutes, exhibiting obvious analgesic and antiinflammatory effects $[167,168]$.

\subsubsection{The Mechanism of Chinese Herbal Medicine in Improving} Blood Rheology and Microcirculation. Some studies have shown that the CPID patients are always under a situation of hypercoagulable blood. TCM defines this state as a blood stasis, and CHMs can accelerate blood circulation and eliminate stasis to improve this situation.

"Fuyanjingheji" (a kind of compound of CHM consisting of Sparganium stoloniferum, Rhizoma Curcuma, Semen Persicae, Fructus Meliae Toosendan, Rhizoma Alismatis, etc.) is used in CPID patients. A clinical research has shown that "Fuyanjingheji" could reduce the whole blood viscosity, blood plasma viscosity, and hematocrit, indicating that the drug can significantly improve the status of thickness, stickiness, and coagulation in blood of CPID patients [169]. "Penyanping," an empirical Chinese herbal compound (consists of Viola yedoensis Makino, Hedyotis diffusa, Forsythia suspensa, Parthenocissus himalayana planch, Salvia miltiorrhiza, etc.), was used to treat CPID patients in Chinese clinic. Clinical research results indicated that "Penyanping" can improve the pelvic hemodynamics indexes, ovarian left arteriopalmus index, bilateral resistance index, maximal speed of left arterial blood flow, and score of time-velocity within CPID patients and prompt the absorption of inflammation [170]. "Manpenzhuyutang" is another Chinese herbal compound (consists of Pollen Typhae, Faeces Trogopterori, Corydalis yanhusuo, Ligusticum chuanxion, Cyperus rotundus, etc.) commonly used to treat CPID patients in Chinese clinic for a long period. One study proved it may improve that the blood rheology indicators in CPID rats model [171]. "Jingangteng Dispersible 
Tablet (JDT)" is a Chinese herbal patent used to treat CPID; the main composition of JDT is Smilax. An experiment indicated that JDT can promote blood circulation, dispel blood stasis, clear away pathogenic heat, and remove the toxin in CPID rat model. After treatment, the whole blood viscosity index significantly decreased, the state of blood stasis was improved, and the efficacy seemed to be dose dependent [172].

\subsubsection{The Mechanism of Chinese Herbal Medicine in Enhanc-} ing Immunity. The body's immune function is a key factor in reducing inflammation. A large number of experimental studies have shown that Chinese herbal compounds may improve the symptoms of immune disorders, thus reducing the inflammations of reproductive organs.

(i) Enhancing the Function of Humoral Immunity. As part of the humoral immune system, IgG, IgM, and IgA play a key role in protecting the body against infection [173]. Determining serum immunoglobulin (Ig) concentration may help estimate humoral immunity function. "Fuke Qianjin tablet," a Chinese herbal patent, whose major ingredients are Philippine Flemingia Root, Radix Zanthoxyli, Root of Cherokee Rose, Andrographitis Paniculata, Radix Codonopsis, and so forth, was widely used to treat various gynecological inflammations. The experiment showed that "Fuke Qianjin tablet" was able to promote the production of IgA, IgG, and IgM in acute pelvic inflammatory disease (APID) rat model, thus improving the immune function and enhancing the antibacterial, anti-inflammatory, and anti-infection effects [174].

(ii) Enhancing the Function of Cellular Immunity. The ratio of CD4/CD8 was lower at the situation of chronic inflammation, which indicated the dysfunction of the immunity [175]. Additionally, TNF is an important cytokine to promote inflammation and has a good effect on the immune system at the initial stage of inflammation, but if it continues to rise, it would make a series of pathophysiological changes. One study showed that the ratios of CD4/CD8 and IL-2 were lower in women with CPID than non-CPID women, while the level of TNF- $\alpha$ was higher, indicating that CPID is closely related to the decline of immunity [176]. A clinical observation found that the ratios of CD4/CD8 and IL-2 were increased, while the level of TNF- $\alpha$ was decreased after treatment with "Penqiangyan granule" for CPID patients and rat model [177, 178]. Another Chinese herbal compound called "Tiedongqingtang" (consists of Ilex purpurea Hassk, Herba Taraxaci, Carthamus tinctorius, Angelica Sinensis, Ligusticum chuanxiong, Radix Paeoniae Rubra, etc.) was reported to improve the level of serum IL-2 in CPID patients, which might be one of the underlying mechanisms in enhancing immune function and improving the symptoms of CPID [179].

2.3. Acupuncture and Moxibustion for the Treatment of Genital Infection. As an important part of CAM, acupuncture and moxibustion, either used alone or in combination, can be an effective treatment for several diseases. The utilization of acupuncture is based on the meridian system: there are some acupoints along the meridian lines, which may have some beneficial effects on a certain disease when stimulated via needling, pressure, or heat [152]. Acupuncture directly acts on the lesion with a short treatment course and a reduced recurrence rate. Data mining of the literature revealed that acupoints on the Ren meridian were commonly selected to treat CPID. Guanyuan (CV4), zigong (EX-CA1), zhongji (RN3), zusanli (ST36), sanyinjiao (SP6), and mingmen (DU4) were the most frequently used for CPID [180]. Ren meridian is one of the eight extra meridians which originates in the lower abdomen. Acupuncture contains a variety of related treatment methods, such as moxibustion, auricular application, and pull cans. Acupuncture has achieved great success in treating gynecological inflammation of the reproductive system since it plays an important role in the anti-inflammatory processes. The mechanism may be due to activating the hypothalamic-pituitary-ovarian axis and the immune system, affecting the nerve-reproductive and endocrine-immune system, dilating blood vessels and lymphatic vessels, accelerating blood circulation, and inhibiting vascular permeability to reduce the inflammatory exudate and accelerate inflammatory exudate absorption [181].

2.3.1. Acupuncture. Acupuncture is the most direct and basic method in this system. It requires using thin metal needles to pierce through skin into certain points to regulate the flow of qi around the whole body [182]. Acupoints are the special position where the "qi" of viscera and meridians intersect and effuse. Stimulating the acupoints can regulate "qi" and blood around the body. A special method of acupuncture puts an absorbable surgical suture (catgut) into the points as a kind of foreign protein. Sutures can therefore continuously stimulate acupoints, thereby allowing the body to produce some abnormal reactions to enhance the phagocytosis of white blood cells, which ultimately strengthens the antiinflammatory effect.

2.3.2. Acupoint Injection with Drugs. As one of the modern therapies, acupoint injection usually requires injecting a drug into acupuncture points to cure certain diseases. The drugs will be absorbed through the subcutaneous tissues and tiny blood vessels. The needles produce positive stimulation at local acupuncture points, which can promote the local blood circulation, elevate the metabolic ability, and ameliorate the pathology while promoting inflammation absorption [183]. Radix Astragali injection contains glycosides, polysaccharides, flavonoids, amino acids, and other chemical components, which has a significant effect on regulating immune function [184]. Clinical studies found that Zusanli (ST36) and Guanyuan (CV4) with Radix Astragali injections have better curative effect in treating CPID [185]. Secretory IgA (S-IgA) is the most important molecule secreted by the mucous in the humoral immune system, and the decreasing of S-IgA in local area is closely related to the increasing susceptibility to genial inflammation [186]. An experimental study showed that Guanyuan (CV4) and Zusanli (ST36) with 
Radix Astragali injection can increase the protein level of SIgA in vaginal washing fluid of the CPID rat model, inhibiting excessive secretion of serum IL- 6 and TNF- $\alpha$. This had a certain effect on the abnormal expression of cytokines, which indicated that local immunity had increased after treatment [187].

2.3.3. The Moxibustion. Moxibustion is a technique that produces heat by burning powdered herbal material at the acupoints. It works at the local skin by stimulating the skin receptors, affecting cellular metabolism, meeting the requirements of oxygen of the local tissue, and promoting the emissions of $\mathrm{CO}_{2}$ [188]. At the same time, moxibustion decreases the viscosity of the muscle fiber cells and accelerates blood circulation and eliminates the inflammation. Moxibustion is often combined with acupuncture and Chinese herbal medicine for treatment and has achieved satisfactory effects. One study made Artemisia argyi, Flos Carthami, Semen Persicae, Paeonia lactiflora, and Radix Aucklandiae into moxa sticks and placed them directly on the acupoints of guanyuan (CV4), zhongji (RN3), zigong (EX-CA1), ciliao (BL32), sanyinjiao (SP6), and zusanli (ST36) to treat CPID. All 38 cases had been cured with clinical symptoms disappearing after 5 periods of treatment, which achieved the desired effect [189].

2.3.4. Acupoint Sticking. Acupoint sticking therapy is a process of making Chinese herbal medicine into different formulations such as pills, powders, and ointments and sticking it on the selected acupoints [190]. It can increase the local drug concentration and make the drug permeating directly on the lesion. Acupoint sticking can stimulate the pelvic nerve and promote blood flow to improve the absorption of inflammation. A clinical study used Herba Asari, Herba Ephedrae, Semen sinapis Rhizoma Corydalis, and so forth to treat CPID in the way of acupoint sticking and showed that the total effective rate was $93.33 \%$ [191].

2.3.5. The Cupping Therapy. Cupping is a TCM therapy that dates back at least 2,000 years and has been applied as a formal modality in hospitals throughout China since 1950 [192]. In this method the negative pressure from cupping is one of the main factors that helps to achieve therapeutic effects. This method can improve the microcirculation and the blood rheology and increase the blood capillary permeability. Additionally, it may prompt inflammatory lesion absorption. Generally, negative pressure can extend local blood vessels to improve microcirculation, accelerate angiogenesis, and promote capillary endothelial cell repair [193]. One study treated 26 cases of CPID by the combination of acupuncture and cupping therapy. Results showed that after 6 months of therapy 20 cases had been cured and 6 cases improved [194].

2.3.6. Others. In addition to the above treatments of acupuncture and moxibustion, there are some other related therapies including auricular therapy, pricking blood therapy, and burning acupuncture though they are not used widely in clinic. In addition, the method of the combination of acupuncture, moxibustion, and physical therapy is frequently used to specifically treat genital infection. One clinical study suggested that the total effective rate was $91.27 \%$ when treating 60 patients with PID via a combination of acupuncture, moxibustion, and ultrashort wave [195].

Acupoints are the most important elements in acupuncture prescription and choosing accurate and appropriate fixed acupoints in the clinic is directly related to the therapeutic effect of acupuncture. In addition, based on the fixed set of acupoints, we can increase or reduce acupoints according to the patient's clinical symptoms, which is also the most common method in clinical treatment. We identify commonly used acupoints for the treatment of PID in clinic (Table 4).

\section{Physiotherapy in Genital Infection}

In recent years, a series of clinical studies has explored the physical therapies used to treat female reproductive tract infections including electrotherapy, magnetic therapy, heat therapy, light therapy, and mud therapy. Physical therapy can be a physical factor used alone and can also be used in combination with two or more physical factors or with the use of drugs, which can enhance the efficacy and shorten the course of treatment. Here we review commonly used physical therapy treatments for gynecological diseases.

3.1. External High Frequency Calorimeter. Microwave hyperthermia can be used for the treatment of chronic pelvic inflammatory disease and vaginitis. With low frequency, long wavelength, and penetrating power, it is difficult to distribute the force generated inside to the rest of the body as almost all is absorbed as heat [196]. The mechanism includes improving the local tissue microcirculation, accelerating the elimination of the inflammatory substances accumulated in the local area, improving pelvic blood circulation and diseased tissue oxygen, increasing the metabolic rate, while reducing the excitability of sensory nerves and interfering with pain impulse conduction, and enhancing the ability of phagocytic cells, thus improving the body's immune function. Clinical studies demonstrated that enema combined with physiotherapy treatment for CPID has been successful and should therefore be adopted. In a clinical study examining physical therapy combined with traditional Chinese medicine retention enema to treat CPID, 32 cases were cured among 40 cases; in the observation group, the total efficiency reached $90 \%$ without any adverse reactions [197].

3.2. Low-Frequency Pulse Therapy. This type of therapy works by acting directly on local nerves and acting indirectly on body fluids, thus enhancing the therapeutic effect of CPID. Electromagnetic waves can adjust muscle contraction rate rising to the maximum point of physiological range and lead drug directly into the local lesion by affecting the magnetic field and maintaining a high local concentration, improving the effect of drug treatment [198]. 
TABLE 4: The list of available acupoints and the frequency of them in treating PID.

\begin{tabular}{|c|c|c|c|}
\hline & Acupuncture point & English name & $\begin{array}{l}\text { The frequency of related points } \\
\text { Totle: } 107\end{array}$ \\
\hline \multirow{7}{*}{ The main points } & Sanyinjiao & SP6 & $N=40$ \\
\hline & Guanyuan & CV4 & $N=38$ \\
\hline & Zhongji & RN3 & $N=36$ \\
\hline & Zusanli & ST36 & $N=28$ \\
\hline & Qihai & CV6 & $N=25$ \\
\hline & Zigong & EX-CA1 & $N=23$ \\
\hline & Guilai & ST29 & $N=20$ \\
\hline \multirow{12}{*}{ The minor points } & Shenshu & BL23 & $N=15$ \\
\hline & Ciliao & BL32 & $N=15$ \\
\hline & Xuehai & SP10 & $N=15$ \\
\hline & Yinlingquan & SP9 & $N=14$ \\
\hline & Shuidao & ST28 & $N=10$ \\
\hline & Taichong & LR3 & $N=4$ \\
\hline & Diji & SP8 & $N=4$ \\
\hline & Daimai & GB26 & $N=4$ \\
\hline & Daheng & SP15 & $N=3$ \\
\hline & Qichong & ST30 & $N=3$ \\
\hline & Baihuanshu & BL30 & $N=3$ \\
\hline & Mingmen & DU4 & $N=3$ \\
\hline
\end{tabular}

3.3. Chinese Medicine Iontophoresis. Traditional Chinese medicine iontophoresis makes use of the principle that similar charges oppose each other and opposite charges attract in direct current (DC). In this way, Chinese medicine ions are able to penetrate into the corresponding lesions in the human body, to achieve the purpose of medical treatment [1]. In recent years, there have been numerous clinical reports about the effect of Chinese medicine iontophoresis treating CPID. In the electric field, Chinese medicine ions penetrate into the focus of infection, playing a dual therapeutic effect of DC and drug treatment [199]. Advantage of this method for CPID is that the drug works directly on the lesion area. The drug ions therefore generate a relatively higher concentration in the lesion area than that of oral drug which enters the blood circulation. Additionally, they have an extended duration, which extends the therapeutic effect at lower dosages compared to oral therapy and while achieving a comparable effect.

\subsection{Ultrashort Wave Combined with Intermediate Frequen-} cy. Ultrashort wave in treating PID shows a good antiinflammatory effect including (i) improving blood and lymph circulation, increasing vascular permeability, alkalinizing the $\mathrm{pH}$ of lesions, eliminating acidosis of local tissue, and promoting lesion organization drying to avoid the damaging effects of tissue edema. Further, it increases the ability of the reticuloendothelial system and white blood cells (WBC) and increases lectin and complement. This also helps to enhance the regeneration process of connective tissue, with good growth of granulation tissue, and thus localized inflammation and wound healing are enhanced. Ultrashort wave electric fields form a bad living environment for bacteria, indirectly inhibiting growth and reproduction of bacterium, and achieve the effect of sterilization, anti-inflammation, and analgesia to PID [200]. Computer intermediate frequency (IF) therapy apparatus is a kind of microcomputer-controlled and low-frequency modulated intermediate frequency current, which has a significant effect on improving local blood circulation, loosening adhesions, and softening the scar and analgesic [201]. Therefore, using ultrashort wave and computer IF in combination creates a synergistic effect, which can ultimately help eliminate pelvic inflammatory exudation and fibrosis. A clinical study demonstrated that the ultrashort wave combined computer IF group was significantly more effective compared to the simple ultrashort wave group [202].

3.5. Ozone Therapy. Clinical studies have shown that ozone treatment is a safe and effective method for female genital infection, especially for vaginal inflammation. Ozone possesses efficient, rapid, and broad-spectrum antiseptic qualities and plays a key role in killing pathogenic microorganisms. Gynecological ozone instrument is based on the technique of low temperature plasma which produces high concentrations of ozone by ionizing the gas molecules in the air. The instrument consists of two parts: one part homogenizes the ozone concentration of an entire portion of the reproductive tract and kills a variety of pathogenic microorganisms in doing so; the other part of the fumigation is to take advantage of the superior bactericidal capacity of ozone, in order to make ozone disperse directly into the area and complete sterilization. Ozone liquid will not 
damage the acid-base balance in the vagina and reverts into pure oxygen rapidly, which will allow the normal growth of vaginal bacteria and inhibit the growth of anaerobic bacteria [203].

\section{Psychological Intervention Therapy}

Many women who suffer from genital infection are at risk of psychological problems including anxiety and depression. Related studies show that psychosocial factors play an important role in the occurrence and development of the disease [204]. Patients with genital inflammation fear repeated course and bear heavy psychological burden for a long time, which may lead to depression, irritability, and anxiety, thereby aggravating the disease. The appropriate psychotherapy may help achieve beneficial results in treating genital infection.

4.1. Support. Expressing concern and understanding for the patient's suffering, explaining each of the issues raised by patients, establishing a good doctor-patient relationship, and obtaining the trust and cooperation from the patients are key to maintaining adequate psychosocial health for women who may be experiencing genital infection.

4.2. Acknowledge. Conducting depression, anxiety, and disease-related lectures-such as teaching patients about the pathogenesis, clinical manifestations, complications, and treatment of the disease-can help educate patients making them mentally confident in overcoming it.

4.3. A Combined Therapy of TCM and Psychological Intervention. Patients who suffer from genital infection are always under the situations of anxiety and depression. Psychological treatment could enhance resilience and positive affect, then improving health and well-being, so psychological treatment combined with TCM not only cures the disease itself but also could provide additional efficacy of improving the quality of life by regulating the psychological situations for those patients undergoing genital infection.

A clinical study indicated that psychological treatment united with Chinese herbal patent was significantly better than traditional therapies for chronic pelvic pain [205]. "Xiaozhengsan" is a traditional Chinese herbal compound (consists of Rhizoma Homalomenae, Zanthoxylum piperitum DC, Cortex Acanthopanacis, Radix Angelicae Dahuricae, Taxillus chinensis, Radix Paeoniae Rubra, etc.) that has been used in treating gynecologic diseases for years; applying Xiaozhengsan externally combined with psychological and behavioral intervention could significantly improve the efficacy and patients' physiological and psychological quality of life [206]. Simple select 164 patients with chronic pelvic inflammatory disease were divided into two groups; the results showed that psychological care stress therapy combined with enema and DC therapy had a significantly greater effect over the control group without psychological care [207].
4.4. Music Therapy. Music care and alternative therapies in clinical applications are becoming increasingly widespread [208]. One study showed that 180 women with pelvic inflammatory disease were randomly divided into a treatment group and a control group. Women in the control group were treated with anti-CPID drugs and pelvic therapy instruments, while women in the treatment group were treated with the same drugs, pelvic therapy instrument, and music therapy. The anxiety scores in the music group significantly decreased when compared to the control group after treatment, and the total average time to improve efficiency and symptoms were significantly better than the control group. Music therapy is a common method of psychological intervention, which has been widely used in the treatment and care of patients in clinical practice $[209,210]$. The majority of patients suffer from anxiety, insomnia, and lack of energy, which seriously affect the rehabilitation of the disease. Music affects the brain in the form of sound waves and stimulates the pituitary gland to release morphine-like substances, which can inhibit pain impulses [211]. As a special psychological treatment, music therapy can improve anxiety, relieve pain, and increase comfort among susceptible patients. Additionally, it is more economical and an easier lifestyle modification for patients to attempt and accept.

\section{Medical Rehabilitation}

Medical rehabilitation gymnastics is a very important content for CPID patients in their remission stage, because it helps regulate the function of the autonomic nervous system, relieve local pain, improve metabolism, and relieve pelvic inflammatory adhesions, thus improving the quality of life of patients with CPID. Studies show that Kung-Fu support is playing a positive role in improving the quality of patients' lives. After accepting pelvic medical rehabilitation treatment for 3 months, scores of each index of CPID patients had increased $[212,213]$, which indicated an improved quality of life score.

\section{Conclusion and Future Directions}

Genital infection is a widespread gynecologic disease, particularly among reproductive age women. If inflammation is left untreated, it will negatively impact the women's immune system, metabolism, and endocrine system. There are additional considerations with pregnant women such as causing intrauterine infection and reproductive tract and neonatal infection, both leading to serious consequences. Numerous clinical studies in vitro and in vivo have shown that TCM may be an effective treatment for genital infection and will not cause tolerance and adverse reactions after prolonged use. Chinese medicines treat genital infection based on the holistic concept and the theory of syndrome differentiation treatment. TCM has achieved the clinical curative effect of antibacterial and anti-inflammatory therapeutics, thus improving both immunity and microcirculation. Chinese medicine has made remarkable achievements in the treatment of genital infection, and this paper reviews select 
effective Chinese herbal compounds that are commonly used. Chinese medicine treatment works through a variety of mechanisms: oral administration, external application, and acupuncture. Whether used as single or incombination therapy can affect how patients may respond to various types of treatment regimens. However, it would be beneficial to conduct large-scale, randomized clinical trials in the future to identify the efficacy of CAM and help substantiate its therapeutic effect.

\section{Conflict of Interests}

The authors declare that there is no conflict of interests regarding the publication of this paper.

\section{Authors' Contribution}

Chenfang Liu and Yuehui Zhang contributed equally to this work and should be considered cofirst authors.

\section{Acknowledgments}

This work was supported by the National Natural Science Fund of China (81273788) and Project of Excellent Innovation Talents by Heilongjiang University of Chinese Medicine.

\section{References}

[1] G. Weiss, L. T. Goldsmith, R. N. Taylor, D. Bellet, and H. S. Taylor, "Inflammation in reproductive disorders," Reproductive Sciences, vol. 16, no. 2, pp. 216-229, 2009.

[2] W. Y. Wu, "Alternative and complementary effect of Chinese medicine in treating malignant tumor," Chinese Journal of Integrated Traditional and Western Medicine, vol. 31, no. 1, pp. 111-114, 2011.

[3] J. Zhou and F. Qu, "Treating gynaecological disorders with traditional Chinese medicine: a review," African Journal of Traditional, Complementary and Alternative Medicines, vol. 6, no. 4, pp. 494-517, 2009.

[4] S. M. Li, J. Chen, and L. S. Chen, "The combination of Shaofuzhuyutang and laparoscopy in the treatment of 124 cases with infertility caused by chronic pelvic inflammatory disease," Fujian Journal of TCM, vol. 44, no. 1, pp. 13-14, 2013.

[5] X. L. Zhu, "Adjusted Shaofuzhuyutangin treating 60 cases with chronic pelvic inflammatory disease," Shanxi Journal of TCM, vol. 34, no. 11, p. 1448, 2013.

[6] L. H. Wu, "Clinical observation on the Shaofuzhuyutangin treating chronic pelvic inflammatory disease," Acta Chinese Medicine and Pharmacology, vol. 38, no. 2, pp. 117-118, 2010.

[7] Y. S. Yin and Y. M. Liu, "Adjusted Shaofuzhuyutang in treating 60 cases with chronic pelvic inflammatory disease of damp-cold retention type," China Modern Medicine, vol. 18, no. 24, pp. 125126, 2011.

[8] S. L. Wang, "The clinical observation on the treatment of 40 cases with chronic pelvic inflammatory diseaseby adjusting Shaofuzhuyutang," Forum on Traditional Chinese Medicine, vol. 27, no. 1, p. 29, 2012.

[9] Y. B. Ding and Z. X. Qian, "Adjusted Shaofuzhuyutang in treating 260 cases with chronic pelvic inflammatory disease,"
Research \& Information on Traditional Chinese Medicine, vol. 7, no. 8, pp. 26-27, 2005.

[10] J. Fu and Y. Xia, “The internal and external therapy of Shaofuzhuyutang for chronic pelvic inflammatory disease," Modern Journal of Integrated Traditional Chinese and Western Medicine, vol. 21, no. 14, pp. 1513-1514, 2012.

[11] L. J. Zhang, "The clinical observation on the treatment of 56 cases with chronic pelvic inflammatory disease of damp-cold retention type," World Latest Medicine Information, vol. 13, no. 3, pp. 198-204, 2013.

[12] H. X. Lu, H. Y. Li, and W. L. Zhao, "The clinical observation on the treatment of 40 cases with chronic pelvic inflammatory diseaseby adjusting Shaofuzhuyutang," Journal of ZheJiang College of Traditional Chinese Medicine, vol. 33, no. 4, pp. 541-542, 2009.

[13] X. W. Zhong, "The clinical observation by ultrasonography onthe treatment of chronic pelvic inflammatory diseaseby adjusting Shaofuzhuyutang," World Health Digest, vol. 9, no. 12, pp. 406-407, 2012.

[14] Y. Gu and K. J. Wang, "The clinical observation of Shaofuzhuyutang in treating chronic pelvic inflammatory disease," The Chinese Journal of Human Sexuality, vol. 20, no. 6, pp. 3839, 2011.

[15] H. F. Guan and X. L. Wang, "Integrated traditional Chinese and Western medicine for the treatment of 50 cases of chronic pelvic inflammation," Modern Traditional Chinese Medicine, vol. 31, no. 5, pp. 17-18, 2011.

[16] W. W. Liu, "Observation of Dangguishaoyaosan for chronic pelvic inflammatory disease belonged to syndrome of humid heat stagnating: a report of 60 cases," Shanxi Journal of Traditional Chinese Medicine, vol. 27, no. 3, pp. 16-17, 2011.

[17] H. Zhang, Y. B. Li, and Z. Jin, "The effect of Dangguishaoyaosan in the treatment of chronic pelvic inflammatory disease," Chinese Journal of Information on TCM, vol. 16, no. 10, pp. 58$59,2009$.

[18] J. J. Wang and Z. D. Sun, "Jiawei Dangguishaoyaosan in the treatment of 56 cases with chronic pelvic inflammatory disease," Shanxi Journal of Traditional Chinese Medicine, vol. 19, no. 2, p. 26, 2003.

[19] S. Q. Ge and L. Liu, "The clinical analysis of effects on treating chronic PID with modification of Guizhifulingtang," China Practical Medicine, vol. 2, no. 12, p. 80, 2007.

[20] S. F. Zhang, "Jiawei Guizhifulingtang in the treatment of chronic pelvic inflammatory disease," Journal of Medical Forum, vol. 26, no. 20, p. 66, 2005.

[21] Y. X. Liu, "The effect of Guizhifulingtang in the treatment of chronic pelvic inflammatory disease," China Modern Medicine, vol. 19, no. 26, pp. 124-125, 2012.

[22] Y. C. Wang and L. P. Liu, "Jiawei Guizhifulingtang in the treatment of 80 cases with chronic pelvic inflammatory disease," Biomagnetism, vol. 5, no. 2, pp. 65-66, 2005.

[23] Q. Wang and A. G. Zeng, "The clinical observation on the treatment of chronic pelvic inflammatory disease by adjusting Danzhixiaoyaosan," Nei Mongol Journal of Traditional Chinese Medicine, pp. 52-53, 2011.

[24] G. X. Liu, "Danzhixiaoyaosan in the treatment of chronic pelvic inflammatory disease," Health World, vol. 2, no. 18, p. 312, 2012.

[25] W. X. Li, "The clinical observation on Chinese herbal compound as adjuvant treatment in 50 cases with chronic pelvic inflammatorydisease," China Health Care \& Nutrition, no. 9, pp. 556-557, 2013. 
[26] L. X. Ding and Y. Q. Meng, "Interventional treatment of vulvovaginal candidiasis 30 cases with Bixieshenshitang," in Proceedings of the 10th National TCM Gynecology Academic Conference, pp. 224-245, 2010.

[27] W. L. Zheng, X. C. Liu, and C. M. Zhao, "Efficacy of Bixieshenshitang combined with fluconazole in the treatment of the complex vulva and vaginal candidiasis," Journal of Guiyang College of Traditional Chinese Medicine, vol. 33, no. 5, pp. 49-50, 2011.

[28] A. Z. Guang, "Integrative medicine in the treatment of 100 cases with vulvovaginal candidiasis," China's Naturopathy, vol. 20, no. 2, pp. 54-55, 2012.

[29] H. X. Gao and W. L. Zheng, "The observation of treating vulvovaginal candidiasis with integrative medicine," Journal of Guiyang College of Traditional Chinese Medicine, vol. 32, pp. 2628, 2010.

[30] L. Zhang and W. L. Zheng, "The observation of integrative medicine in the treatment of 60 cases with severe vulvovaginal candidiasis," Journal of North Pharmacy, vol. 8, no. 1, pp. 78-79, 2011.

[31] Q. Z. Deng, "Observation of Wandaitang in the treatment of cervicitis," China Foreign Medical Treatment, no. 11, pp. 45-46, 2008.

[32] J. Liu, "Clinical observation on colpitis mycotica treated with Wan-dai decoction," Journal of Chengdu University of Traditional Chinese Medicine, vol. 32, no. 1, pp. 31-32, 2009.

[33] X. L. Jia, "Adjusted Longdanxiegantang in the treatment of 50 cases of gonorrhea vaginitis," Jiangxi Journal of Traditional Chinese Medicine, no. 2, pp. 129-130, 1996.

[34] Z. Wu and Z. X. Huang, "Rectal administration with improved Hongtengtang in the treatment of chronic pelvic inflammatory disease," Modern Journal of Integrated Traditional Chinese and Western Medicine, vol. 14, no. 4, pp. 444-445, 2005.

[35] W. H. Feng, Z. K. Wang, and Y. S. Jiang, "Clinical research on targeted treatment with Hongtengtang for pelvic inflammatory disease," Modern Journal of Integrated Traditional Chinese and Western Medicine, vol. 19, no. 26, pp. 3283-3284, 2010.

[36] M. Y. Zhou, "Clinical observation of rectal administration with Hongtengtang on the treatment of pelvic inflammation disease," Chinese and Foreign Medical Research, vol. 9, no. 4, pp. 49-50, 2011.

[37] G. F. Zeng, Y. Yang, and J. F. Liu, "Clinical observation of rectal administration with Hongtengtang on the treatment of chronic pelvic inflammation disease," China Practical Medicine, vol. 8, no. 3, p. 144, 2013.

[38] X. M. Qiu, "Observation and nursing care of rectal administration with Hongtengtang on the treatment of chronic pelvic inflammation disease," Jilin Medical Journal, vol. 33, no. 35, pp. 7810-7811, 2012.

[39] H. Q. Miao, "Hongtengtang in the treatment of chronic pelvic inflammation disease," Nei Mongol Journal of Traditional Chinese Medicine, no. 5, pp. 65-66, 2012.

[40] C. H. Zhang and Y. Du, "Traditional Chinese medicine in the treatment of 48 cases with pelvic inflammatory disease," Modern Traditional Chinese Medicine, vol. 29, no. 5, p. 19, 2009.

[41] X. H. Qian, "Rectal administration with Chinese Herbal Medicine in the treatment of pelvic inflammatory disease," Clinical Journal of Traditional Chinese Medicine, vol. 22, no. 5, pp. 439-440, 2010.

[42] H. F. Zhang, "Hongtengtang in the treatment of 35 cases with pelvic inflammatory disease," Medical Information, vol. 24, no. 7, pp. 4804-4805, 2011.
[43] L. Yuan, "Observation of treatment of trichomonas vaginitis with the combination of Wandaitang orally and Kushentang externally," Chinese Community Doctors, vol. 14, no. 3, pp. 193194, 2012.

[44] S. P. Yang, "An observation on treating 120 cases of vaginitis patients by TCM internal using and extrernal washing," Clinical Journal of Chinese Medicine, vol. 3, no. 11, pp. 85-88, 2011.

[45] M. Jiang, "The observation of integrative medicine in the treatment of trichomonas vaginitis," Chinese Manipulation and Rehabilitation Medieiqne, vol. 2, no. 7, p. 182, 2011.

[46] F. L. Lu and X. S. Zhao, "The observation of integrative medicine in the treatment of 35 cases with candida vaginitis," Modern Journal of Integrated Traditional Chinese and Western Medicine, vol. 14, no. 1, p. 64, 2005.

[47] X. Yan and J. Yu, "The observation of integrative medicine in the treatment of 60 cases of trichomonas vaginitis," Journal of Modern Clinical Medicine, vol. 36, no. 1, pp. 30-31, 2010.

[48] M. Wang, X. Liu, and X. L. Wang, "Clinical research of the treatment of fungal vaginitis by washing with Longdanxiegantang," Guangming Journal of Chinese Medicine, vol. 27, no. 9, pp. 18061807, 2012.

[49] F. E. Chen, "Longdanxiegantang in the treatment of 152 cases of trichomonas vaginitis," Henan Traditional Chinese Medicine, vol. 21, no. 3, p. 48, 2001.

[50] Y. Yang and H. X. Fan, "The clinical and experimental study on the curative effect of Longdan Xiegan Pill(Decoction) on Bacterial Vaginosis (BV)," The Journal of Medical Theory and Practice, vol. 18, no. 11, pp. 1277-1279, 2005.

[51] H. Shi and J. Xie, "Longdan Xiegan decoction combined with azithromycin vaginal administration treating female genital ureaplasma urealyticum infection," Research of Integrated Traditional Chinese and Western Medicine, vol. 4, no. 1, pp. 1-3, 2012.

[52] Y. L. Chen, J. Q. Zhang, and X. B. Wang, "Analgesic effect of pelvic inflammation enema Decoction on abirritation of pelvic inflammatory disease and influence of immune function," China Medical Herald, vol. 10, no. 6, pp. 88-90, 2013.

[53] H. H. Zhu and J. L. Fu, "Observation of treatment of chronic pelvic inflammatory disease with the combination of rectal administration andphysiotherapy," Chinese Journal of Clinical Rational Drug Use, vol. 6, no. 1, p. 21, 2013.

[54] M. Wang, X. Liu, and X. W. Wang, "Clinical research on Long Dan Xie Gantang in treating fungal vaginitis," Guangming Journal of Chinese Medicine, vol. 27, no. 9, pp. 1806-1807, 2012.

[55] Q. Huang and H. M. Wang, "Observation and nursing care of traditional Chinese medicine treatment for 100 cases of fungal vaginitis," China Medical Herald, vol. 5, no. 9, pp. 69-78, 2008.

[56] Y. Wang, Y. H. Cai, and L. Ai, "Vaginal administration in the treatment of 80 cases with chronic pelvic inflammatory disease," China Journal of Chinese Materia Medical, vol. 13, no. 2, p. 39, 1998.

[57] S. Y. Zhang, J. Jiang, M. X. Guo, and S. Y. Wang, "External application of Chinese medicine in the treatment of 28 cases with chronic pelvic inflammatory disease," Shandong Medical Journal, vol. 51, no. 9, pp. 102-103, 2011.

[58] H. Y. Xiang, L. B. Hou, and S. H. Huang, "Pharmacodynamic studies on the effect of Jingangteng dispersible tablets for chronic pelvic inflammatory disease," Journal of Chinese Medicinal Materials, vol. 30, no. 4, pp. 449-452, 2007.

[59] Y. Ma, Y. Q. Luo, and L. Y. Song, "Pharmaceutical screening of the effective fraction from Smilax for treatment of chronic pelvic inflammatory disease," Journal of Southern Medical University, vol. 33, no. 1, pp. 145-149, 2013. 
[60] J. Wang, "The observation of comfrey oil in the treatment of 52 cases of candidiasis," Chinese Journal of Modern Drug Application, vol. 3, no. 13, pp. 94-95, 2009.

[61] F. Z. Meng, "Wild chrysanthemum suppository in the treatment of 40 cases with pelvic inflammatory disease," Guangming Journal of Chinese Medicine, vol. 18, no. 108, p. 35, 2003.

[62] D. Zhao, Q. Ding, and Y. Xiao, "Progress of researches onpatrinia," Guiding Journal of Traditional Chinese Medicine and Pharmacy, vol. 15, no. 10, pp. 76-78, 2009.

[63] Q. L. Li, H. Li, and C. X. Guo, "Treatment of chronic pelvic inflammation by Houttuyniacordata seal off (a clinical observation of 500 cases)," China Journal of Modern Medicine, vol. 15, no. 23, pp. 3633-3634, 2005.

[64] H. H. Zhou, T. X. Zhou, and S. H. Geng, "The research progress of Houttuynia cordata," Medical Information, vol. 24, no. 8, pp. 4125-4125, 2011.

[65] J. Y. Zhang, "Clinical observation on the treatment of 40 cases of chronic pelvic inflammatory disease by adjusting Xianglengtang," Gansu Journal of Traditional Chinese Medicine, vol. 13, no. 3, pp. 4-6, 2000.

[66] Y. Shi, "Yirenfuzibaijiangsan in the treatment of 40 cases of chronic pelvic inflammatory disease," Journal of Practical Traditional Chinese Medicine, vol. 17, no. 9, pp. 14-15, 2001.

[67] X.-Q. Yao and H.-E. Yang, "Clinical observation on integrated traditional Chinese and Western medicine therapy in treating patients with pelvic inflammatory disease: a report of 28 cases," Journal of Chinese Integrative Medicine, vol. 4, no. 2, pp. 199-201, 2006.

[68] W. G. Zhan and J. Chang, "The clinical experience of treating chronic pelvic inflammatory disease with Fuyankangfang by professor Z. X. Pei," China Practical Medicine, vol. 7, no. 11, pp. 227-228, 2012.

[69] Y. P. Jin, "The combination of acupuncture, moxibustion and Chinese Herbal Medicine in the treatment of 36 cases with chronic pelvic inflammatory disease," Zhejiang Journal of Integrated Traditional Chinese and Western Medicine, vol. 13, no. 12, pp. 772-783, 2003.

[70] C. L. Cui, "Fukexiaoyanfang in the treatment of 55 cases with chronic pelvic inflammatory disease," Guangming Journal of Chinese Medicine, vol. 24, no. 8, pp. 1472-1473, 2009.

[71] P. Zhao, "The analysis of effect on Shuganyangxuetang in the treatment of 58 cases with chronic pelvic inflammatory disease," Guide of China Medicine, vol. 7, no. 19, pp. 76-77, 2009.

[72] C. H. Zhao and Z. Q. Zhang, "The observation of Hongtengdahuangdanpitang in the treatment of 85 cases with chronic pelvic inflammatory disease," Clinical Journal of Traditional Chinese Medicine, vol. 21, no. 6, pp. 541-542, 2009.

[73] J. Y. Lu, "Improved Buzhongyiqitang in the treatment of 53 cases with chronic pelvic inflammatory disease," Chinese Archives of Traditional Chinese Medicine, vol. 26, no. 7, pp. 1580-1581, 2008.

[74] F. G. Guo, "Qingresanyutang in the treatment of 78 cases with chronic pelvic inflammatory disease," Sichuan Journal of Traditional Chinese Medicine, vol. 25, no. 7, pp. 76-77, 2007.

[75] L. M. Yang, "Lichongtang in the treatment of chronic pelvic inflammatory disease," China's Naturopathy, vol. 17, no. 9, p. 37, 2009.

[76] S. J. Ge and S. Ge, "Syndrome differentiation and treatment in the treatment of 82 cases with chronic pelvic inflammatory disease," Shanxi Journal of Traditional Chinese Medicine, vol. 24, no. 5, p. 388, 2003.
[77] Y. N. Lin, "Danshenpugongtang in the treatment of 65 cases with chronic pelvic inflammatory disease," China's Naturopathy, vol. 15, no. 4, p. 21, 2007.

[78] J. P. Jiang, "Observation of jiaweierhaofang for chronic pelvic inflammatory disease belonged to syndrome of humid heat stagnating," Clinical Journal of Traditional Chinese Medicine, vol. 22, no. 6, pp. 504-505, 2010.

[79] J. L. Kuang and Y. F. Xi, "The observation of Penyanfang in the treatment of 30 cases with chronic pelvic inflammatory disease (syndrome of humid heat stagnating)," Journal of New Chinese Medicine, vol. 40, no. 4, pp. 57-58, 2008.

[80] B. Li, "Observation of treating 30 cases with chronic pelvic inflammatory disease belonged to syndrome of humid heat stagnating with the rule of clearing heat-resolving dampnessremoving-stasis-arresting leukorrhea," Hebei Journal of Traditional Chinese Medicine, vol. 30, no. 5, p. 482, 2008.

[81] P. Lu, F. H. Wu, and Y. L. Yu, "Gufupenyufang in the treatment of 80 cases with chronic pelvic inflammatory disease belonged to syndrome of cold-damp stagnation," Jiangxi Journal of Traditional Chinese Medicine, vol. 39, no. 311, pp. 40-41, 2008.

[82] Q. M. Wang, “Observation of Jiaweiwandaitang in treating bacterial vaginosis belonged to the type of damp abundance due to splenic asthenia," Beijing Journal of Traditional Chinese Medicine, vol. 30, no. 5, pp. 384-385, 2011.

[83] B. Q. Shen, Z. Chen, and J. L. Huang, "Observation of the treatment of 27 cases with recurrent candida vaginitis by combining Wandaitang and Clotrimazole suppositories for external use," Journal of New Chinese Medicine, vol. 39, no. 12, pp. 52-53, 2007.

[84] Y. F. Wang, "The observation of integrative medicine in the treatment of 60 cases of trichomonas vaginitis," Jiangsu College of TCM, vol. 22, no. 4, p. 45, 2005.

[85] Z. C. Li, "Zhimeitang in the treatment of 65 cases of indolent fungal vaginitis," Journal of Sichuan of Traditional Chinese Medicine, vol. 29, no. 9, p. 87, 2011.

[86] L. Zhang and W. L. Zheng, "The observation of integrative medicine in the treatment of 60 cases of severe vulvovaginal candidiasis," Journal of North Pharmacy, vol. 8, no. 1, pp. 78-79, 2011.

[87] H. P. Zhu, "An observation on treating 66 cases of fungal vaginitis patients by TCM internal using and external washing," Journal of Practical Traditional Chinese Medicine, vol. 23, no. 2, p. 84, 2007.

[88] P. Y. Zhu, "The combination of internal and external therapy of 113 cases with Senile vaginitis," China's Naturopathy, vol. 18, no. 3, pp. 46-47, 2010.

[89] Y. C. Huang, "An observation on treating 46 cases of trichomonas vaginitis patients by TCM internal using and external steaming," Shanxi Journal of Traditional Chinese Medicine, vol. 28, no. 7, p. 678, 2007.

[90] J. Liu, "The observation of Wandaitang in the treatment of recurrent fungal vaginitis," Journal of Chengdu University of TCM, vol. 32, no. 1, pp. 31-32, 2009.

[91] X. X. Li, H. Ye, and X. Y. Liu, "The clinical analysis of Zinizhidaifang in the treatment of 125 cases with recurrent bacterial vaginosis," Journal of Sichuan of Traditional Chinese Medicine, vol. 29, no. 11, pp. 86-87, 2011.

[92] Y. Sun, "The combination of internal using and external washing in the treatment of trichomonas vaginitis," China Practical Medicine, vol. 6, no. 16, pp. 143-144, 2011. 
[93] Y. P. Wang and R. X. Chen, “The combination of internal using and external washing with Chinese Herbal Medicine in the treatment of fungal vaginitis," Guide of China Medicine, vol. 10, no. 10, pp. 271-272, 2012.

[94] X. L. Gao, "Clinical observation on the treatment of 50 cases of chronic cervicitis by adjusting xiaoyaosan," Journal of Traditional Chinese Medicine, vol. 47, no. 10, pp. 792-793, 2006.

[95] Y. L. Bai and J. P. Li, "Experience of Tounongsan in the treatment of 20 cases chronic cervicitis postoperative," China and Foreign Medical Journal, vol. 6, no. 2, pp. 48-49, 2008.

[96] S. L. Li and Y. J. Cui, "Clinical observation on the treatment of 50 cases of chronic cervicitis by combining Shenlingbaizhusan and washing with Metronidazole," Chinese Practical Journal of Rural Doctor, vol. 11, no. 11, p. 31, 2004.

[97] L. X. Wang, “The experience of applying Siwutang in the treatment of chronic cervicitis after the treatments of the microwave instrument," Jilin Journal of Traditional Chinese Medicine, vol. 27, no. 6, p. 30, 2007.

[98] M. L. Zhang, "The combination of internal and external therapy of 84 cases with chronic cervicitis," Forum on Traditional Chinese Medicine, vol. 19, no. 1, pp. 39-40, 2004.

[99] Z. X. Hou, "Wuweixiaoduyin in the treatment of 31 cases with cervicitis infected by mycoplasma," Shandong Journal of Traditional Chinese Medicine, vol. 29, no. 2, p. 98, 2010.

[100] W. H. Li and H. Liu, "The combination of rectal administration andexternal application on the treatment of 80 cases with chronic pelvic inflammation disease," Yunnan Journal of Traditional Chinese Medicine and Materia Medica, vol. 32, no. 5, p. 98, 2011.

[101] N. Z. Du, L. S. Geng, and X. H. Song, "Clinical observation of rectal administration with Chinese Herbal Medicine on the treatment of 160 cases with chronic pelvic inflammation disease," Chinese Journal of Family Planning, vol. 16, no. 12, pp. 751-752, 2008.

[102] L. H. Duan, "Clinical observation of rectal administration with Chinese Herbal Medicine on the treatment of 53 cases with chronic pelvic inflammation disease," Endemic Diseases Bulletin, vol. 21, no. 4, p. 123, 2006.

[103] S. Q. Yuan, "Clinical observation of rectal administration with Chinese Herbal Medicine on the treatment of 30 cases with chronic pelvic inflammation disease," Shanxi Journal of Traditional Chinese Medicine, vol. 23, no. 5, p. 418, 2002.

[104] L. Y. Zhou, "Clinical observation of rectal administration with Hongtengtang on the treatment of 78 cases with chronic pelvic inflammation disease," The Medical Journal of Industrial Enterprise, vol. 20, no. 1, p. 13, 2007.

[105] Q. Y. Fan, "Clinical observation of rectal administration with Jiaweiermiaosan on the treatment of 56 cases with chronic pelvic inflammation disease," Guangming Journal of Chinese Medicine, vol. 2, no. 27, p. 83, 2007.

[106] P. Li, "Clinical observation of Fukangwan for external in the treatment of 65 cases with chronic pelvic inflammation disease," Journal of Henan University of Chinese Medicine, vol. 22, no. 6, p. 59, 2007.

[107] Y. X. Yan and Q. X. Wang, "Clinical observation of rectal administration with Dahuangmudantang on the treatment of chronic pelvic inflammation disease," Modern Journal of Integrated Traditional Chinese and Western Medicine, vol. 17, no. 1, p. 56, 2008.

[108] R. M. Qu and J. X. Fan, "Clinical observation of external therapy by promoting blood circulation and removing blood stasis for chronic pelvic inflammation disease," Journal of Practical Medical Techniques, vol. 12, no. 12, p. 3707, 2005.

[109] B. Lin, "Chinese medicine iontophoresis in the treatment of 126 cases with infertility caused by chronic pelvic inflammation disease," China's Naturopathy, vol. 13, no. 1, p. 25, 2005.

[110] Y. H. Kang, S. Y. Li, and Y. L. Li, "Clinical observation of rectal administration with Guixiongyantaoyin on the treatment of 60 cases with chronic pelvic inflammation disease," Shandong Journal of Traditional Chinese Medicine, vol. 24, no. 4, p. 223, 2005.

[111] Z. Y. Fu, Z. Q. Li, G. Han et al., "Rectal administration with Fufanghuzhangxiaoyantang in the treatment of 50 cases with chronic pelvic inflammation disease," Chinese Journal of Integrated Traditional and Western Medicine, vol. 21, no. 3, p. $237,2001$.

[112] G. X. Zhang, "The combination of external application and rectal administration with traditional herbal medicine on the treatment of 86 cases with chronic pelvic inflammation disease," Journal of Sichuan of Traditional Chinese Medicine, vol. 18, no. 9, p. 34, 2000.

[113] D. Q. Lin and Z. Y. Li, "Western medicine and rectal administration with traditional herbal medicine on the treatment of 22 cases with chronic pelvic inflammation disease," Shanxi Journal of Traditional Chinese Medicine, vol. 26, no. 5, pp. 390-391, 2005.

[114] S. Y. Du, "Rectal administration with traditional herbal medicine on the treatment of 20 cases with chronic pelvic inflammation disease," Liaoning Journal of Traditional Chinese Medicine, vol. 32, no. 3, p. 227, 2005.

[115] L. M. Zhao, "External application with traditional herbal medicine on the treatment of 30 cases with chronic pelvic inflammation disease," Journal of External Therapy of Traditional Chinese Medicine, vol. 13, no. 6, p. 13, 2004.

[116] C. J. Jiang, "Chinese medicine iontophoresiso in the treatment of chronic pelvic inflammation disease," Journal of Practical Traditional Chinese Medicine, vol. 16, no. 12, p. 30, 2000.

[117] Q. Y. Xu and X. Q. Huang, "The clinical observation of herbal lotion in treatment of vaginitis," Journal of Community Medicine, vol. 9, no. 5, pp. 32-33, 2011.

[118] L. H. Zhao, "The clinical observation of herbal lotion in treatment of vaginitis," Guide of China Medicine, vol. 8, no. 28, pp. 24-25, 2010.

[119] C. X. Shang and H. Y. Zhang, "The clinical analysis of Chinese Herbal Medicine on the treatment of 1000 cases with vaginitis," China Practical Medicine, vol. 5, no. 28, pp. 135-136, 2010.

[120] D. H. Yu, "The clinical observation of vaginal lavage with Chinese Herbal Medicine in thetreatment of trichomonas vaginitis," China Foreign Medical Treatment, vol. 28, no. 3, p. 125, 2009.

[121] Y. L. Nie, "Redaisan in thetreatment of 300 cases with recurrent fungal vaginitis," Journal of Sichuan of Traditional Chinese Medicine, vol. 29, no. 11, p. 88, 2011.

[122] X. Zou, "The efficiency analysis of the treatment of 300 cases with recurrent fungal vaginitis," Modern Medicine and Health, vol. 27, no. 23, pp. 3621-3622, 2011.

[123] M. Wang, X. Liu, and X. L. Wang, "The clinical research of washing with Longdanxiegantang in the treatment of fungal vaginitis," Guangming Journal of Chinese Medicine, vol. 27, no. 9, pp. 1806-1807, 2012.

[124] X. Yan and J. Yu, "The clinical observations of treating trichormonal vaginitis with traditional Chinese and Western medicine," Journal of Modern Clinical Medicine, vol. 36, no. 1, pp. 30-31, 2010. 
[125] G. R. Kong and L. Zhu, "Zhimeizhiyang lotion in the treatment of 200 cases with Candida vaginitis," Journal of Practical Traditional Chinese Medicine, vol. 23, no. 1, pp. 46-47, 2007.

[126] Q. Liu, "Jieduqingyinye in the treatment of 150 cases with vulvovaginitis," Hebei Journal of Traditional Chinese Medicine, vol. 29, no. 1, p. 31, 2007.

[127] C. Y. Yang, "The clinical observations of Chinese Herbal Medicine in the treatment of 96 cases with Fungal vaginitis," Medical Journal of Chinese People's Health, vol. 20, no. 2, p. 115, 2008.

[128] M. Q. Zhan, "The clinical observation of Shechuangzi lotion combined with Western medicine in the treatment of 256 cases with vaginitis," Journal of Community Medicine, vol. 6, no. 8, p. 47, 2008.

[129] Y. R. Tang, "The clinical observation of Yixiling in the treatment of vulvovaginitis and vaginitis," Modern Journal of Integrated Traditional Chinese and Western Medicine, vol. 16, no. 7, pp. 892893, 2007.

[130] B. P. Deng, "Steaming and washing with Chinese Herbal Medicine in the treatment of 62 cases with candida vaginitis," Journal of Emergency in Traditional Chinese Medicine, vol. 16, no. 10, p. 1204, 2007.

[131] J. M. Wang and X. Y. Pang, "The analysis of Chinese Herbal Medicine in the treatment of 50 cases with fungal vaginitis," Chinese Journal of Misdiagnostics, vol. 8, no. 2, pp. 475-476, 2008.

[132] D. Lei and L. H. Mu, "Preparation and clinical application of Kushenshehuangsan," Journal of External Therapy of Traditional Chinese Medicine, vol. 16, no. 6, p. 57, 2007.

[133] Y. Liu, "Chinese Herbal Medicine powder in the treatment of 101 cases with chronic cervicitis," China Practical Medicine, vol. 28, no. 5, pp. 146-147, 2010.

[134] Z. D. Zhao, "The clinical observation of Gongjingyan powder in the treatment of 78 cases with chronic cervicitis," Yunnan Journal of Traditional Chinese Medicine and Materia Medica, vol. 25, no. 3, p. 59, 2004.

[135] G. Z. Zhang and C. L. Duan, "The external use of Chinese Compound Xiguashuang in the treatment of 80 cases with cervical erosion," China's Naturopathy, vol. 11, no. 1, p. 25, 2003.

[136] H. B. Wu, "The clinical research on Huangjieshebeisan in the treatment of chronic cervicitis," Lishizhen Medicine and Materia Medica Research, vol. 22, no. 3, p. 3, 2011.

[137] X. L. Zhu and Y. Ma, "The clinical observation of Erchakuiyangsan in the treatment of chronic cervicitis," Fujian Journal of Traditional Chinese Medicine, vol. 34, no. 5, pp. 6-7, 2003.

[138] X. M. Zhu, "Chibaihuangzigao in the treatment of 116 cases with simplex cervical erosion," Chinese Journal of Rural Medicine and Pharmacy, vol. 14, no. 5, p. 47, 2007.

[139] X. Q. Yu and Z. P. Yao, "Washing with Kubaitang in the treatment of cervical erosion," Shandong Journal of Traditional Chinese Medicine, vol. 21, no. 4, p. 242, 2002.

[140] M. L. Wen, "The clinical observation of Huangliansanin the treatment of 96 cases with chronic cervicitis," Journal of Aerospace Medicine, vol. 20, no. 10, p. 145, 2009.

[141] Z. C. Zuo, G. D. Zhou, and J. Y. Wang, “The clinical observation of Gongjingyankangshuan in the treatment of 76 cases with chronic pelvic inflammation disease," Journal of Bethune Military Medical College, vol. 9, no. 5, pp. 325-326, 2011.

[142] H. Li, "The efficiency research on traditional Chinese medicine in the treatment of chronic cervicitis and vaginitis," Clinical Journal of Chinese Medicine, vol. 3, no. 20, pp. 103-104, 2011.
[143] L. X. Xia and X. Q. Ma, "Gongjingyan powder in the treatment of cervical erosion," China's Naturopathy, vol. 15, no. 5, p. 18 , 2007.

[144] J. T. Zhang and R. L. Jia, "Shajiziyou suppositories in the treatment of 86 cases with cervical erosion," Modern Traditional Chinese Medicine, vol. 26, no. 5, pp. 45-46, 2006.

[145] T. B. Wang, "The clinical observation of Gongjingyan powder in the treatment of 78 cases with chronic cervicitis," Liaoning Journal of Traditional Chinese Medicine, vol. 25, no. 3, p. 59, 2004.

[146] X. C. Wang and R. J. Zhao, "External therapy with Chinese Herbal Medicine in the treatment of 97 cases with chronic cervicitis," China's Naturopathy, vol. 12, no. 6, p. 18, 2004.

[147] W. L. Li and L. J. Chen, "The clinical observation of comfrey oil in the treatment of chronic cervicitis," Chinese Community Doctors, vol. 11, no. 16, p. 14, 2009.

[148] L. H. Tang and C. M. Wang, "The clinical observation of Chinese Herbal Medicine in the treatment of chronic cervicitis," Chinese Journal of Primary Medicine and Pharmacy, vol. 14, no. 11, pp. 1931-1932, 2007.

[149] K. Vermani and S. Garg, "Herbal medicines for sexually transmitted diseases and AIDS," Journal of Ethnopharmacology, vol. 80, no. 1, pp. 49-66, 2002.

[150] H. Azimi, M. Fallah-Tafti, M. Karimi-Darmiyan, and M. Abdollahi, "A comprehensive review of vaginitis phytotherapy," Pakistan Journal of Biological Sciences, vol. 14, no. 21, pp. 960966, 2011.

[151] R. L. Sweet, "Treatment of acute pelvic inflammatory disease," Infectious Diseases in Obstetrics and Gynecology, vol. 2011, Article ID 561909, 13 pages, 2011.

[152] J. C. Longhurst, "Defining meridians: a modern basis of understanding," Journal of Acupuncture and Meridian Studies, vol. 3, no. 2, pp. 67-74, 2010.

[153] Y. H. Ben, Y. L. Ding, and H. Y. Li, "Bacteriostasis of penyanqing granule on common pathogenic bacteria," Journal of Changchun University of Traditional Chinese Medicine, vol. 24, no. 4, p. 368, 2008.

[154] P. Qiu, X. B. Sheng, and S. H. Qiu, "Extracts from Smilax by different techniques for the treatment of chronic pelvic inflammatory disease," Chinese Traditional and Herbal Drugs, vol. 41, no. 12, pp. 2046-2047, 2012.

[155] T.-T. Fu, J.-Y. Wu, L. Wang et al., "Study on the Chinese herbal formula for treatment of vaginitis and the antimicrobial activity in murine models," Journal of Chinese Medicinal Materials, vol. 29, no. 9, pp. 931-936, 2006.

[156] K. S. Hsu and H. Y. Kao, “ $\beta$-transducin repeat-containing protein 1 ( $\beta$-TrCP1)-mediated silencing mediator of retinoic acid and thyroid hormone receptor (SMRT) protein degradation promotes tumor necrosis factor $\alpha$ (TNF $\alpha$ )-induced inflammatory gene expression," The Journal of Biological Chemistry, vol. 288, no. 35, pp. 25375-25386, 2013.

[157] R. F. Liu, X. N. Yang, and B. Q. Li, "The clinical and experiment study of penqiangyan granule on the treatment of chronic pelvic inflammatory disease," Journal of Shandong University of TCM, vol. 27, no. 6, pp. 422-425, 2003.

[158] Y. Sun, L. H. Hou, and X. K. Wu, "The influence of Yan Ting enema on TNF- $\alpha$ and ICAM-1 in tubal of rats with inflammatory infertility," Lishizhen Medicine and Materia Medica Research, vol. 19, no. 11, pp. 2681-2683, 2008.

[159] P. Wu, L. Zhang, and D. J. Zhang, "The study on relationship of pharmacological mechanism of anti-inflammatory traditional 
Chinese Medicine and its effect on promoting relieving inflammation," Chinese Journal of Pathophysiology, vol. 21, no. 8, p. 1658, 2005.

[160] S. B. Wei, Y. Q. Yao, and Y. Wang, "The influence of penyangkangshuan on the expression of NF-KB, Caspase-3, MMP-2 in the endometrial cells of rats with pelvic inflammatory disease," Chinese Journal of Information on Traditional Chinese Medicine, vol. 13, no. 3, pp. 31-32, 2006.

[161] C. Q. Yuan, “The structure and function of caspase," Foreign Medicine, vol. 24, no. 3, p. 146, 2002.

[162] I. Krolikiewicz-Renimel, T. Michel, E. Destandau et al., "Protective effect of a Butea monosperma (Lam.) Taub. flowers extract against skin inflammation: antioxidant, anti-inflammatory and matrix metalloproteinases inhibitory activities," Journal of Ethnopharmacology, vol. 148, no. 2, pp. 537-543, 2013.

[163] P. F. M. Verhaak, J. J. Kerssens, J. Dekker, M. J. Sorbi, and J. M. Bensing, "Prevalence of chronic benign pain disorder among adults: a review of the literature," Pain, vol. 77, no. 3, pp. 231239, 1998.

[164] F. A. Beebe, R. L. Barkin, and S. Barkin, "A clinical and pharmacologic review of skeletal muscle relaxants for musculoskeletal conditions," American Journal of Therapeutics, vol. 12, no. 2, pp. 151-171, 2005.

[165] M. von Korff, A. Kolodny, R. A. Deyo, and R. Chou, "Long-term opioid therapy reconsidered," Annals of Internal Medicine, vol. 155, no. 5, pp. 325-328, 2011.

[166] C. Elder, C. Ritenbaugh, M. Aickin et al., "Reductions in pain medication use associated with traditional Chinese medicine for chronic pain," The Permanente Journal, vol. 16, no. 3, pp. 1823, 2012.

[167] J. Y. Chen, Q. Tang, and L. Zhu, "The observation of the effect of anti-inflammation and analgesic of Jin Gangteng tablets," Sichuan Journal of Physiological Sciences, vol. 22, no. 1, pp. 16-18, 2000.

[168] X. S. Shu, Z. H. Gao, and X. L. Yang, "Anti-inflammatory and anti-nociceptive activities of Smilax china L. aqueous extract," Journal of Ethnopharmacology, vol. 103, no. 3, pp. 327-332, 2006.

[169] C. Y. Zhao, L. L. Hou, and R. Shen, "The clinic research on Fuyanjingheji in the treatment of 120 cases with chronic pelvic inflammatory disease," Jiangsu Journal of Traditional Chinese Medicine, vol. 25, no. 6, pp. 15-17, 2004.

[170] Y.-X. Huang and S.-Q. Xue, "Analysis on the significance of pelvic hemodynamics in efficacy evaluation of TCM treatment for chronic pelvic inflammation," Chinese Journal of Integrated Traditional and Western Medicine, vol. 27, no. 10, pp. 932-934, 2007.

[171] S. M. Feng and W. F. Sun, "The process of research on mechanism of treating chronic pelvic inflammatory disease with the rule of promoting blood circulation and removing blood stasis," Military Medical Journal of South China, vol. 24, no. 6, pp. 499-504, 2010.

[172] H. Y. Xiang, Y. L. Wang, L. B. Hou et al., "Effect of Jingangteng dispersible tablet on chronic pelvic inflammatory disease in the blood rheology and pathological histology of rat," Journal of Modern Food and Pharmaceuticals, vol. 17, no. 2, pp. 38-41, 2007.

[173] H. W. Schroeder Jr. and L. Cavacini, "Structure and function of immunoglobulins," Journal of Allergy and Clinical Immunology, vol. 125, no. 2, pp. S41-S52, 2010.

[174] X. Li, J. S. Guo, and Z. Y. Shi, "Effect of Fuke Qianjin tablets on inflammatory cytokines in blood serum in rats with chronic pelvic inflammatory disease," Chinese Journal of Experimental Traditional Medical Formulae, vol. 19, no. 10, pp. 225-228, 2013.
[175] D. Dicker, M. A. Salook, D. Marcoviciu, M. Djaldetti, and H. Bessler, "Role of peripheral blood mononuclear cells in the predisposition of obese individuals to inflammation and infection," Obesity Facts, vol. 6, no. 2, pp. 146-151, 2013.

[176] Q. Sa, J. Woodward, and Y. Suzuki, "IL-2 produced by CD8 ${ }^{+}$ immune $\mathrm{T}$ cells can augment their IFN- $\gamma$ production independently from their proliferation in the secondary response to an intracellular pathogen," Journal of Immunology, vol. 190, no. 5, pp. 2199-2207, 2013.

[177] R.-F. Liu and X.-N. Yang, "Effect of Penqiangyan Granule on the immune function of patients with chronic pelvic inflammatory disease of blood-stasis and Shen-deficiency syndrome type," Chinese Journal of Integrated Traditional and Western Medicine, vol. 27, no. 9, pp. 841-843, 2007.

[178] R. F. Liu, X. N. Yang, and B. Q. Li, "Clinical and experimental study on chronic pelvic inflammatory disease treated with Penqiangyan granules," Journal of Shangdong University of Traditional Chinese Medicine, vol. 27, no. 6, pp. 422-425, 2003.

[179] Y. Zhong, "The influence of Tiedongqingtang on the level of IL2 in patients with chronic pelvic inflammatory disease," Journal of North Pharmacy, vol. 10, no. 3, p. 31, 2013.

[180] M. L. Chen and D. L. Ju, "The recent advances of acupuncture and moxibustionin in the treatment of chronic pelvic inflammatory disease," Shanghai Journal of Acupuncture and Moxibustion, vol. 30, no. 11, pp. 795-798, 2011.

[181] Y.-P. Xue, S.-B. Zhang, and T. Gao, “Observation on therapeutic effect of chronic prostatitis treated mainly by warming needle moxibustion," Chinese Acupuncture \& Moxibustion, vol. 26, no. 5, pp. 335-336, 2006.

[182] S. J. Birch and R. L. Felt, Understanding Acupuncture, Churchill Livingstone, New York, NY, USA, 1999.

[183] Q. Wang, J.-X. Xiong, and W.-Y. Pan, "Observation on treatment of chronic pelvic inflammatory with point injection combined with ultra-laser radiation," Chinese Acupuncture \& Moxibustion, vol. 26, no. 9, pp. 614-616, 2006.

[184] X. L. Zhang and L. P. Zhang, "The research of effect of Leguminosae on the immune system," Chinese Journal of Information on Traditional Chinese Medicine, vol. 42, no. 4, p. 111, 2011.

[185] Y. Zhang and P. Chen, "The influence of acupoint injection of astragalus parenteral solution on Rheology and plasma inflammatory cytokines of patients with chronic pelvic inflammatory disease," Fujian Journal of Traditional Chinese Medicine, vol. 42, no. 4, pp. 13-14, 2011.

[186] P. C. Giraldo, A. K. Gonçalves, and J. Eleutério Junior, "Secretory immunoglobulin A: a protective factor in the genital mucosa," The Brazilian Journal of Infectious Diseases, vol. 10, no. 4, pp. 232-234, 2006.

[187] L. F. Chen, X. F. Cai, and Z. L. Wang, "Effects of Huangqiacupoint injection on immunity function in rats with chronic pelvic inflammatory disease," Chinese Archives of Traditional Chinese Medicine, vol. 25, no. 11, pp. 2321-2324, 2007.

[188] Z. Fang, "Warmed-needle acupuncture treat 65 cases of chronic pelvic inflammatory disease," Acupuncture and Moxibustion and Massage, vol. 27, no. 2, pp. 107-108, 2008.

[189] X. Q. Shang, "Moxibustion with Chinese Herbal Medicine in treating chronic pelvic inflammatory disease," Chinese Acupuncture and Moxibustion, vol. 21, no. 5, p. 274, 2001.

[190] Y. P. Yuan, "Recent clinical studies on Acupoint sticking therapy," Journal of Clinical Acupuncture and Moxibustion, vol. 20, no. 12 , pp. 53-55, 2004. 
[191] J. F. Xu, "Sanfu-Acupoint sticking therapy in the treatment of 30 cases with pelvic inflammatory disease due to damp-cold retention," Zhejiang Journal of Traditional Chinese Medicine, vol. 44, no. 6, p. 452, 2009.

[192] H. Cao, X. Li, and J. Liu, "An updated review of the efficacy of cupping therapy," PLoS ONE, vol. 7, no. 2, Article ID e31793, 2012.

[193] S. Cui and J. Cui, "Progress of researches on the mechanism of cupping therapy," Acupuncture Research, vol. 37, no. 6, pp. 506510, 2012.

[194] X. Y. Cai, "The combination of acupuncture andcupping therapytreat 26 cases of chronic pelvic inflammatory disease," Shanghai Journal of Acupuncture and Moxibustion, vol. 28, no. 12, p. 726, 2009.

[195] X. Xia, "Treat 60 cases of pelvic inflammatory disease by the method of combination of acupuncture, moxibustion and ultrashort wave," Misdiagnosis of China, vol. 8, no. 3, pp. 666$667,2008$.

[196] S. J. Wang, W. J. Tang, and S. F. Gong, "The observation of effect on treating pelvic inflammatory disease withexternal high frequency calorimeter," Chinese Journal of Convalescent Medicine, vol. 19, no. 7, p. 626, 2010.

[197] L. Li, "The combination of physiotherapy and rectal administration in the treatment of pelvic inflammatory disease," Modern Journal of Integrated Traditional Chinese and Western Medicine, vol. 19, no. 36, pp. 4711-4712, 2010.

[198] F. Qian, D. Z. Zeng, and S. F. Li, “The adjuvant effect of electromagnetic low-frequency vibration therapeutic apparatus on chronic pelvic inflammatory disease," Modern Medical Journal, vol. 37, no. 6, pp. 414-416, 2009.

[199] L. Sun and C. Y. Dong, "Treat 128 cases of pelvic inflammatory disease by the method of combination of Chinese medicine iontophoresis and Western medicine enema," Modern Journal of Integrated Raditional Chinese and Western Medicine, vol. 20, no. 8, p. 986, 2011.

[200] C. F. Zhang, "The clinical analysis of physical therapy for chronic pelvic inflammatory disease," International Medicine and Health Guidance News, vol. 12, no. 11, p. 27, 2006.

[201] Y. Wu, S. P. Qi, J. Wu, M. Chai, H. Shen, and Z. Ma, "Two physical factors on treatment chronic pelvic infection," Journal of Dalian Medical University, vol. 27, no. 1, p. 66, 2005.

[202] Y. L. Ding, "Effect of treating chronic pelvic inflammation with ultrashort wave and computerized modulated medium frequency," Anhui Medical Journal, vol. 30, no. 9, pp. 1078-1079, 2009.

[203] X. Z. Peng, "Ozone therapy in treating women's vaginitis," China Practical Medicine, vol. 5, no. 27, pp. 140-141, 2010.

[204] Q. Yan, "The role of psychoneuroimmunology in personalized and systems medicine," Methods in Molecular Biology, vol. 934, pp. 3-19, 2012.

[205] Y. Wang, "Treating 120 cases of chronic pelvic pain by the method of combination of psychological therapy and Kangfuxiaoyanshuan," Liaoning Journal of Traditional Chinese Medicine, vol. 33, no. 1, p. 65, 2006.

[206] B. Chen, "Investigation of psychological-behavioral interventions in treating sequelae of pelvic inflammatory disease," Medicine and Philosophy, vol. 29, no. 4, p. 71, 2008.

[207] J. M. Wang, J. J. Chen, and T. Xia, "Application of psychological stress measures in the treatment care of chronic pelvic inflammatory disease," Chinese Journal of Ethnomedicine and Ethnopharmacy, vol. 19, no. 12, pp. 232-232, 2010.
[208] X. Y. Chen, "Effect and clinical application of music care," Journal of Practical Nursing, vol. 17, no. 4, pp. 50-51, 2001.

[209] S. P. Hu and Z. X. Yang, "Progress of researches on music therapy in Japan," Foreign Medical Sciences, vol. 19, no. 3, pp. 118-119, 2000.

[210] X. P. Wu and B. M. Ji, "The role, status and application of music therapy in nursing," Chinese Journal of Practical Nursing, vol. 5, no. 6, pp. 38-39, 1989.

[211] Y. S. He, W. X. Zeng, and S. Y. Huang, "Music therapy in rehabilitation of patients with chronic pelvic inflammatory disease," International Medicine and Health Guidance News, vol. 12, no. 23, pp. 36-37, 2006.

[212] A. M. Wang, Y. Y. Chen, and J. L. Qiao, "Pelvic medical rehabilitation exercises on the quality of life of patients with chronic pelvic inflammatory disease," Maternal and Child Health Care of China, vol. 24, no. 6, pp. 834-835, 2009.

[213] G. L. Fu and Y. H. Shen, "Pelvic exercises in the emotional outcome of patients with secondary infertility of chronic pelvic inflammatory disease," in Proceedings of the National Integrated Medicine Development Strategy Seminar, p. 335, 2011. 


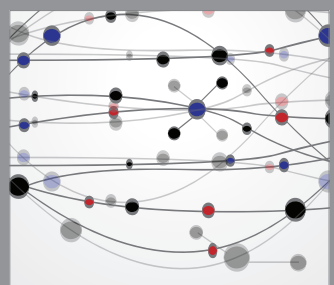

The Scientific World Journal
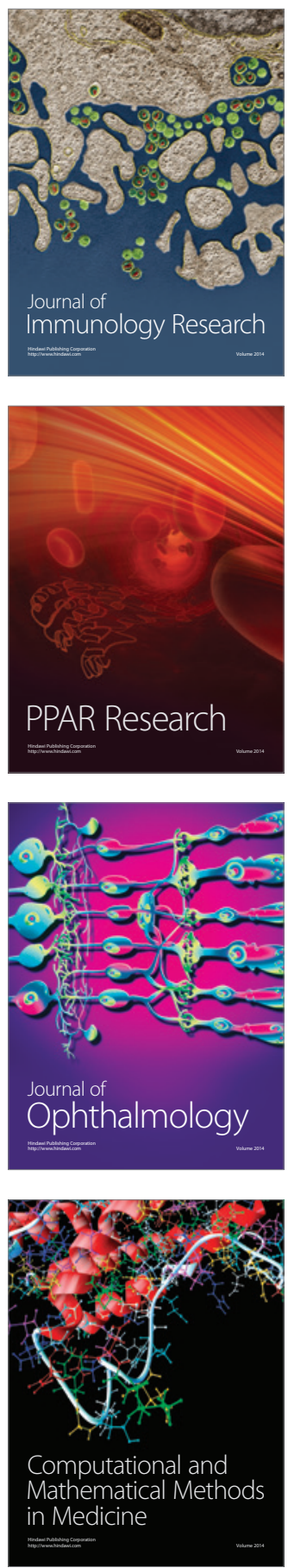

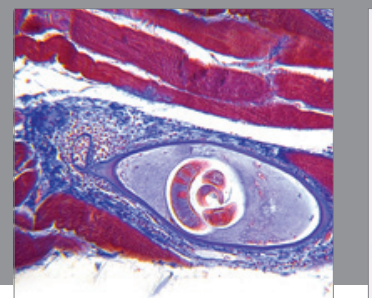

Gastroenterology

Research and Practice
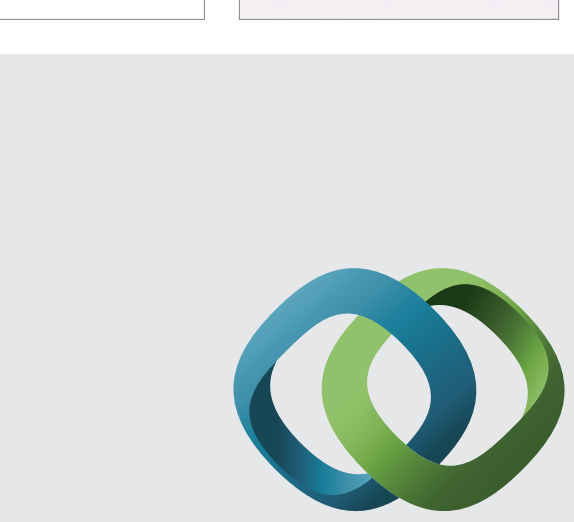

\section{Hindawi}

Submit your manuscripts at

http://www.hindawi.com
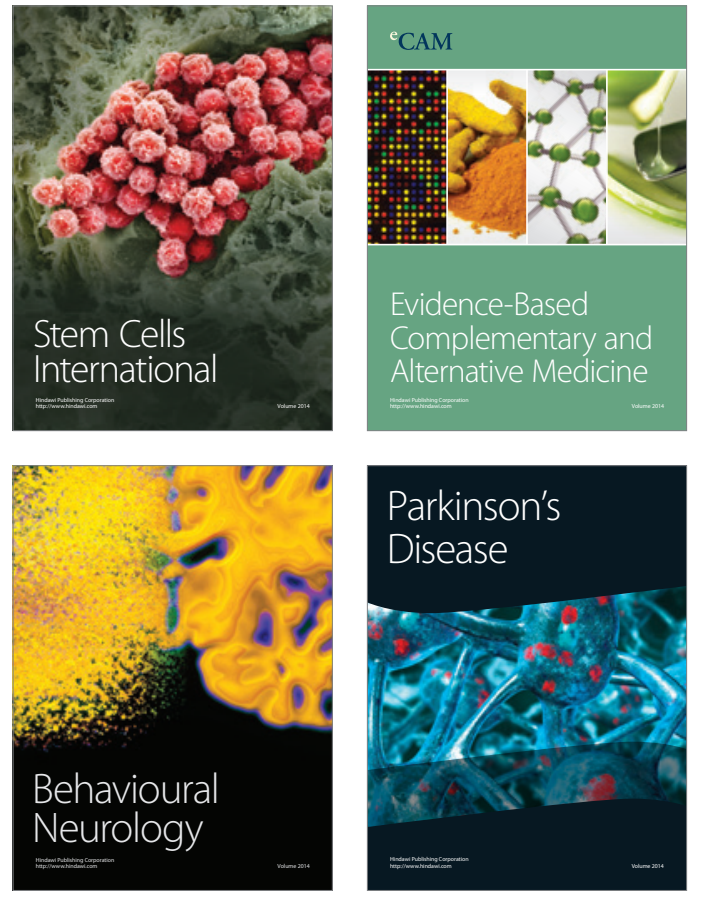
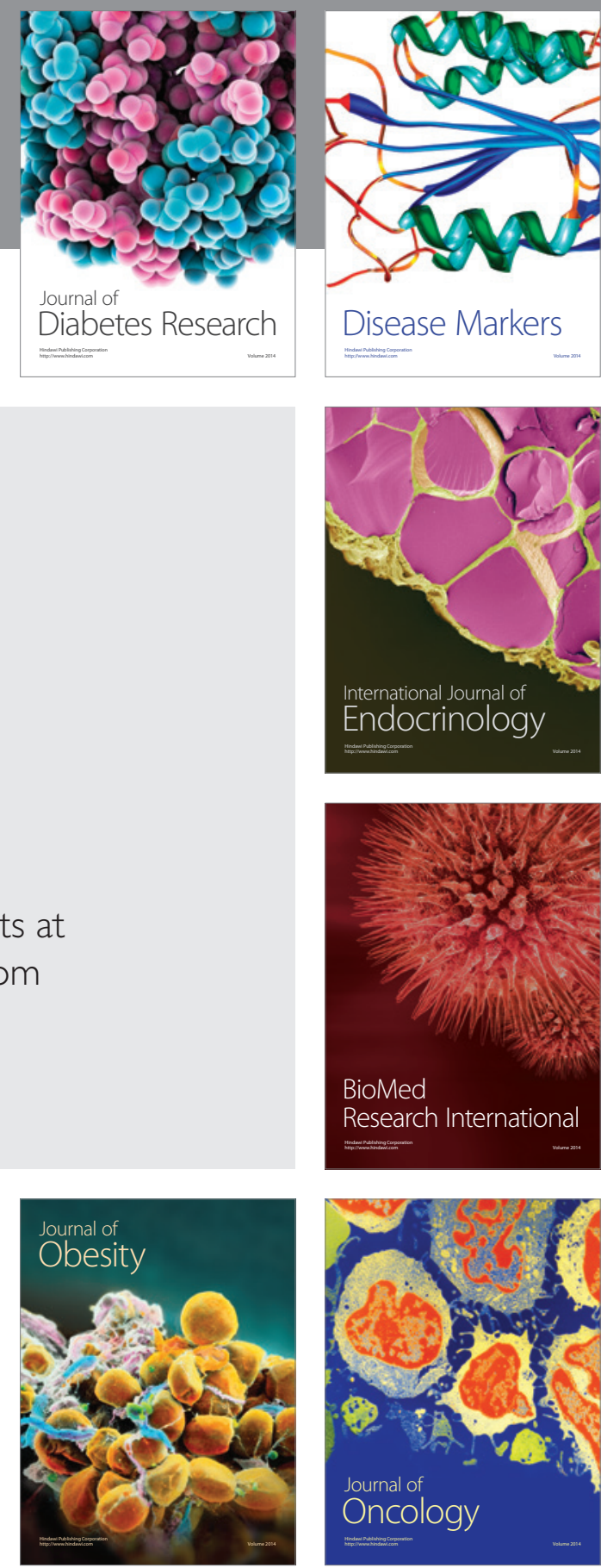

Disease Markers
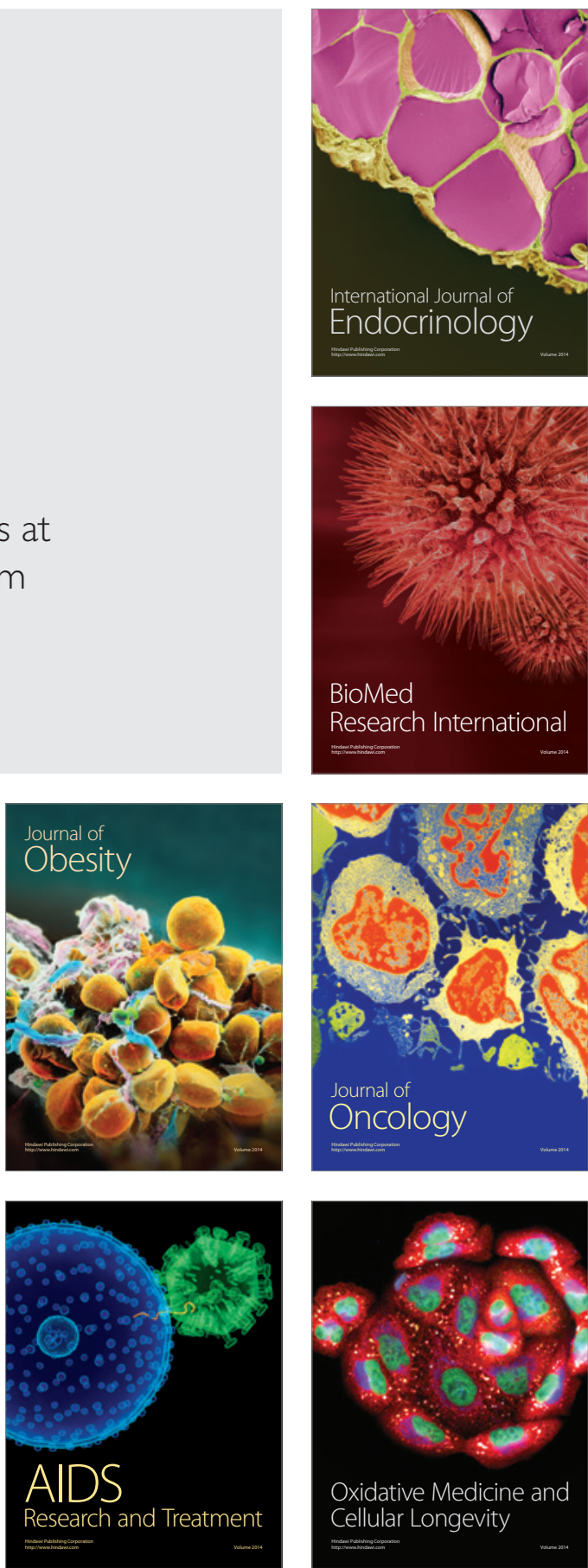